\title{
Neural oscillations track the maintenance and proceduralization of novel instructions
}

\author{
Silvia Formica ${ }^{\mathrm{a}, *}$, Carlos González-García ${ }^{\mathrm{a}}$, Mehdi Senoussi ${ }^{\mathrm{a}}$, Marcel Brass ${ }^{\mathrm{a}, \mathrm{b}}$ \\ ${ }^{a}$ Department of Experimental Psychology, Ghent University, Belgium \\ ${ }^{\mathrm{b}}$ School of Mind and Brain/Department of Psychology, Humboldt Universität zu Berlin, Germany
}

\section{A R T I C L E I N F O}

\section{Keywords:}

Attention

Cognitive control

EEG oscillations

Instruction implementation

Motor preparation

\begin{abstract}
A B S T R A C T
Humans are capable of flexibly converting symbolic instructions into novel behaviors. Previous evidence and theoretical models suggest that the implementation of a novel instruction requires the reformatting of its declarative content into an action-oriented code optimized for the execution of the instructed behavior. While neuroimaging research focused on identifying the brain areas involved in such a process, the temporal and electrophysiological mechanisms remain poorly understood. These mechanisms, however, can provide information about the specific cognitive processes that characterize the proceduralization of information. In the present study, we recorded EEG activity while we asked participants to either simply maintain declaratively the content of novel S-R mappings or to proactively prepare for their implementation. By means of time-frequency analyses, we isolated the oscillatory features specific to the proceduralization of instructions. Implementation of the instructed mappings elicited stronger theta activity over frontal electrodes and suppression in mu and beta activity over central electrodes. On the contrary, activity in the alpha band, which has been shown to track the attentional deployment to task-relevant items, showed no differences between tasks. Together, these results support the idea that proceduralization of information is characterized by specific component processes such as orchestrating complex task settings and configuring the motor system that are not observed when instructions are held in a declarative format.
\end{abstract}

\section{Introduction}

One peculiar aspect of human cognitive flexibility involves the ability to convert complex symbolic instructions into novel behaviors, even in the absence of prior practice (Cole, Laurent, \& Stocco, 2013). Recent experimental evidence and theoretical proposals have put forward a serial heuristic model in which instructions are first encoded and maintained in a declarative format before being transformed into an actionoriented format (i.e., procedural representation) that allows executing the instructed task (Brass, Liefooghe, Braem, \& De Houwer, 2017). Neuropsychological and behavioral evidence supports a dissociation between 'knowing' the content of an instruction and 'doing' the instructed cognitive or motor action, suggesting that maintaining its declarative content is insufficient to implement the instructed task, and that additional procedural codes are needed for optimal performance (Bhandari \& Duncan, 2014; Duncan, Emslie, Williams, Johnson, \& Freer, 1996; Liefooghe, Wenke, \& De Houwer, 2012; Milner, 1963; Wenke, Gaschler, Nattkemper, \& Frensch, 2009). Such immediate reformatting seems to occur only when the instruction is prepared for implementation, and not when participants merely maintain the information in a declarative manner, without the intention to implement (Liefooghe et al., 2012).

A growing number of fMRI studies has primarily focused on revealing which brain regions support the implementation of novel task sets (Demanet et al., 2016; González-García, Arco, Palenciano, Ramírez, \& Ruz, 2017; Hartstra, Kühn, Verguts, \& Brass, 2011; Hartstra, Waszak, \& Brass, 2012; Palenciano, González-García, Arco, \& Ruz, 2019; Ruge \& Wolfensteller, 2010) and, more recently, on characterizing implementation-specific neural representations (Bourguignon, Braem, Hartstra, De Houwer, \& Brass, 2018; González-García, Formica, Wisniewski, \& Brass, 2019; Muhle-Karbe, Duncan, De Baene, Mitchell, \& Brass, 2017; Palenciano, González-García, Arco, Pessoa, \& Ruz, 2019; Ruge, Schäfer, Zwosta, Mohr, \& Wolfensteller, 2019). These results consistently point towards a crucial role of frontoparietal regions, and in particular of the prefrontal cortex (PFC). Similarly, they provide evidence for distinct neural mechanisms supporting implementing versus memorizing novel instructions (Brass et al., 2017). However, it is still not clear to what extent declaratively representing information and proceduralization involve processes that overlap or differ qualitatively. Crucially, investigating the oscillatory dynamics of instruction implementation would allow to decompose the specific spectral components that are

\footnotetext{
* Corresponding author.

E-mail address: silvia.formica@ugent.be (S. Formica).
} 
involved in proceduralizing S-R mappings into action-oriented codes, and to provide insights on their temporal unfolding. This would increase our understanding of the functional significance of such processes, complementing the knowledge on localization and representational format provided by fMRI research.

Specifically, we reasoned that both declarative maintenance and proactive proceduralization of novel instructions likely engage certain shared processes, such as selective attention towards the instructed content, whereas differences in brain activity should reflect the reformatting process that is specific to the implementation of the instruction. On the one hand, changes in the attentional focus are revealed by activity in the alpha frequency range $(8-14 \mathrm{~Hz}$ ) (Hari \& Salmelin, 1997; Klimesch, Sauseng, \& Hanslmayr, 2007; Senoussi, Moreland, Busch, \& Dugué, 2019; van Ede, 2017). Modulations of posterior alpha band oscillations are a well-established mechanism associated with endogenous orienting of spatial attention and suppressing competition from distractors, allowing for the enhancement of task-relevant information (Bonnefond \& Jensen, 2012; Jensen \& Mazaheri, 2010; Sauseng et al., 2005). A substantial body of evidence reports decrease of alpha amplitude over posterior parietal regions contralateral to the attended spatial location, indicating mechanisms of orienting attention both to salient stimuli in the perceptual space and towards internally held representations (Capilla, Schoffelen, Paterson, Thut, \& Gross, 2014; Gould, Rushworth, \& Nobre, 2011; Mok, Myers, Wallis, \& Nobre, 2016; Myers, Walther, Wallis, Stokes, \& Nobre, 2015; Poch, Campo, \& Barnes, 2014; Poch, Carretie, \& Campo, 2017; Rihs, Michel, \& Thut, 2007, 2009; Rohenkohl \& Nobre, 2011; Schneider, Mertes, \& Wascher, 2016; Thut, Nietzel, Brandt, \& Pascual-Leone, 2006; van Dijk, Schoffelen, Oostenveld, \& Jensen, 2008; Wallis, Stokes, Cousijn, Woolrich, \& Nobre, 2015). Additionally, posterior alpha power has been observed to increase with the number of items relevant after attentional selection in a WM task (Jensen, 2002), also following retrospective selection (Manza, Hau, \& Leung, 2014; Poch et al., 2017; Poch, Valdivia, Capilla, Hinojosa, \& Campo, 2018). Therefore, we reasoned that alpha dynamics involved in the selection of internally maintained instructions should be independent of whether the task requires their implementation or mere maintenance.

On the other hand, we hypothesized that instruction implementation relies on a specific set of processes that result in the reformatting of the declarative content into action-oriented code. Therefore, we expected some oscillatory features to be specifically associated with proceduralization. First, the proactive reformatting of the instruction into a proceduralized action-bound representation should involve the allocation of cognitive control to allow the binding of stimulus and response. Ample evidence has associated control-related top-down mechanisms with larger theta (3-7 Hz) amplitude over mid-frontal scalp electrodes (Cavanagh \& Frank, 2014; Cohen \& Donner, 2013). Recent computational models attribute to theta a central role in orchestrating longrange neural communication and in binding task-relevant information (Senoussi et al., 2020; Verbeke \& Verguts, 2019; Verguts, 2017). At the same time, studies showed increased theta power for WM manipulation (Itthipuripat, Wessel, \& Aron, 2013; Onton, Delorme, \& Makeig, 2005). Therefore, we hypothesized stronger mid-frontal theta power during preparation for instruction implementation compared to maintenance, reflecting the proactive reformatting of the S-R mapping into a proceduralized action-bound representation. Nevertheless, behavioral evidence suggests that only a small set of mappings can be proceduralized and maintained in such format prior to the moment of their actual implementation, hinting at a limit in the number of novel instructions that can be proceduralized at once (Liefooghe et al., 2012). As we expect theta power to track the occurrence of such reformatting, we reasoned that theta dynamics should not differ from declarative maintenance when the number of mappings to implement exceeds capacity limits.

Finally, we reasoned that proactively recoding the instruction for execution, and not merely maintaining it, should induce the preparation of the instructed motor plan (Everaert, Theeuwes, Liefooghe, \&
De Houwer, 2014; Meiran, Pereg, Kessler, Cole, \& Braver, 2015a). Therefore, we looked at suppression in beta $(15-30 \mathrm{~Hz})$ and $\mathrm{mu}(8-12 \mathrm{~Hz})$ frequency bands over central electrodes, oscillatory features traditionally associated with motor preparation and motor imagery (Cheyne, 2013; Pineda, 2005; Rhodes, Gaetz, Marsden, \& Hall, 2018; Schneider, Barth, \& Wascher, 2017; Tzagarakis, West, \& Pellizzer, 2015). We expected this modulation to be larger while preparing to implement the instructions, thus when an action-bound procedural representation is available, compared to the declarative maintenance of the mappings. Again, we predicted such difference to be reduced with a higher number of instructions, due to a limit in the number of action plans that can be prepared and maintained ready simultaneously.

To test our hypotheses, we recorded EEG activity while participants performed a task that encouraged a declarative maintenance of novel S-R mappings for recognition (Memorization task), and an Implementation task, intended to prompt their proactive reformatting for execution. In both cases, after the presentation of four mappings at the beginning of the trial, a retro-cue selected a subset of them as potential targets for the subsequent recognition or execution task. We reasoned that brain activity during the Cue-Target interval (CTI) would reflect the two-steps action described in a recent model of information prioritization in WM (Myers, Stokes, \& Nobre, 2017). According to Myers and colleagues (2017), attention needs to be internally oriented towards the selected items, and the prioritized representations can then be reformatted into a behavior-optimized state. In the context of instruction implementation, the task-optimized representation is a procedural, action-bound code of the S-R mapping. On the other hand, successful maintenance is optimally achieved by means of a declarative representation of stimulus and response that does not entail any action plan. Therefore, during the CTI of the two tasks, we expected analogous features associated with attentional orienting, but instances of reformatting to be larger in preparation for instruction implementation.

\section{Materials and methods}

\subsection{Participants}

Thirty-nine participants took part in the experiment $\left(M_{\text {age }}=21.74\right.$, $S D=4.50,33$ females) and received 30 euros as compensation. Sample size was not computed a priori: we aimed for thirty-five participants, which is on the upper bound of the sample size of studies investigating similar constructs (de Vries, Van Driel, Karacaoglu, \& Olivers, 2018; Schneider et al., 2017; van Ede, Chekroud, Stokes, \& Nobre, 2019a). All participants had normal or corrected-to-normal vision and thirty-three reported to be right-handed. Data from two participants were discarded due to low task performance (individual mean accuracy exceeded by 2.5 standard deviations the group mean accuracy in one of the two tasks and/or accuracy in response to catch trials in one of the tasks was below $60 \%$ ); data from two additional participants were discarded following visual inspection because of excessive noise in the EEG recordings, resulting in a final sample size of thirty-five participants. All participants gave their informed consent prior to the beginning of the experiment, in accordance with the Declaration of Helsinki and the protocols of Ghent University.

\subsection{Materials}

The same set of stimuli was used as in previous studies on instruction implementation (Formica, González-García, \& Brass, 2020; GonzálezGarcía, Formica, Liefooghe, \& Brass, 2020). It consisted of 1550 pictures, grouped in two macro categories: animate (non-human animals) and inanimate (vehicles and musical instruments) (Brady, Konkle, Alvarez, \& Oliva, 2013; Brodeur, Guérard, \& Bouras, 2014; Griffin, Holub, \& Perona, 2007; Konkle, Brady, Alvarez, \& Oliva, 2010). All images had their background removed, were centered in a $200 \times 200$ pixels square 


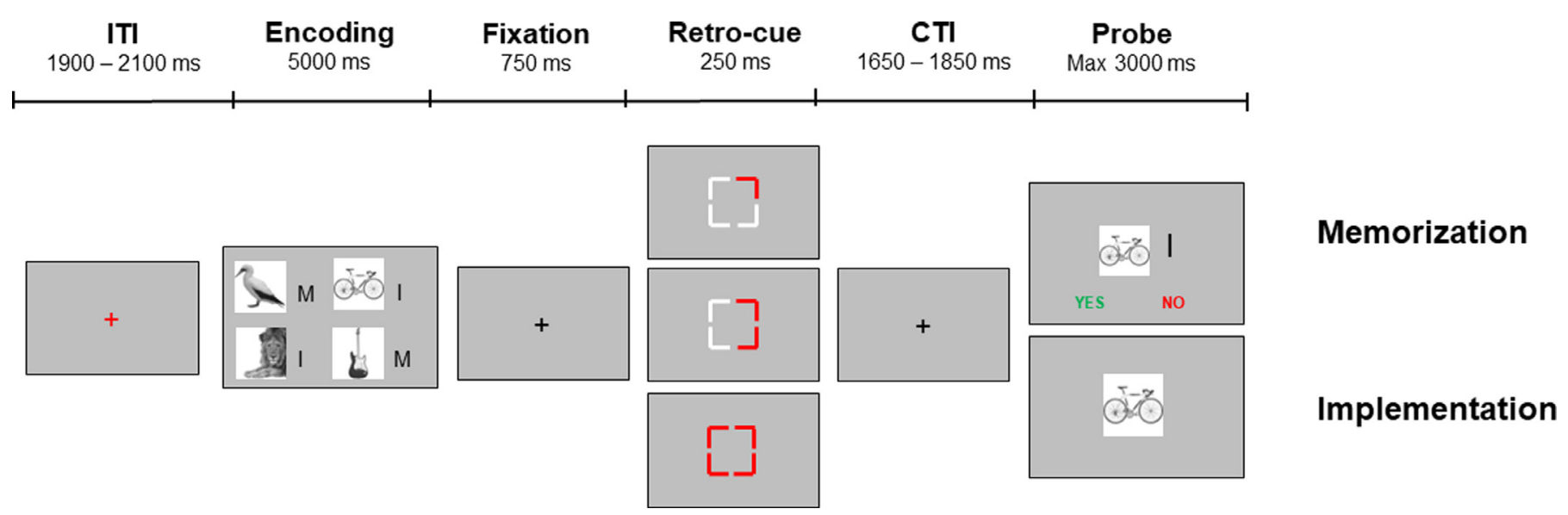

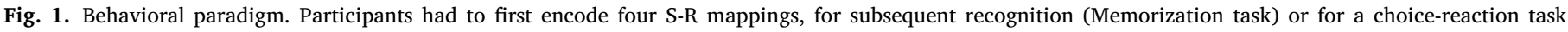

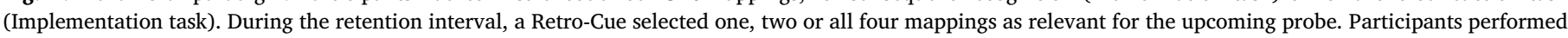
the two tasks separately in a block design.

and were converted to grayscale. Stimuli presentation and response collection were performed using Psychopy toolbox (Pierce, 2007).

\subsection{Experimental design}

Participants performed the two tasks (from now on "Implementation task" and "Memorization task") in a blocked fashion during one single session, and the order of the two tasks was counterbalanced between participants. The structure of the trials for the two tasks was identical up to the presentation of the target (Fig. 1). Each trial started with a red fixation cross presented for $2000 \mathrm{~ms}( \pm 100 \mathrm{~ms}$, jittered) signaling the inter-trial interval and allowing participants to blink if needed, followed by a white fixation cross for $250 \mathrm{~ms}$. Next, the encoding screen containing four S-R mappings (arranged in two rows) appeared for $5 \mathrm{~s}$. Mappings consisted of a new image associated with a bimanual response: "index" referred to both index fingers (keys $r$ and i) and "middle" referred to both middle fingers (keys e and o). These bilateral responses were used instead of the more traditional left and right options to avoid automatic motor activations elicited by the mere presentation of lateralized response words (Bundt, Bardi, Abrahamse, Brass, \& Notebaert, 2015). Out of the four presented mappings, two contained images of animals and two images of inanimate objects. Each image-response combination was presented only once during the whole duration of the experiment. The rationale of this choice was to maximize WM demands and minimize the retrieval of practiced S-R mappings from long-term memory (Meiran, Pereg, Kessler, Cole, \& Braver, 2015b). After a 750 ms delay, which is considered an interval sufficiently long to discard a contribution of iconic memory (Souza \& Oberauer, 2016), the retro-cue appeared and remained on the screen for $250 \mathrm{~ms}$. This consisted of a square presented centrally, with one, two or four (i.e., neutral retro-cue) colored corner(s) ${ }^{1}$. The number of selected mappings is the second crucial factor in our design, namely Load. Participants were instructed that the retro-cue signaled which mapping(s) could be probed, with $100 \%$ validity (except in the case of catch trials, see below). Importantly, when the retro-cue selected two mappings, these were always on the same side of the screen. The subsequent cue-target interval (CTI) had a jittered duration, lasting on average $1750 \mathrm{~ms}( \pm 100 \mathrm{~ms})$. This interval largely exceeds the $300-500 \mathrm{~ms}$ considered necessary for retro-cues to benefit performance (Souza \& Oberauer, 2016) and sufficiently long to

\footnotetext{
1 The physical properties of the retro-cue could potentially produce a combination of exogenous and endogenous orienting of attention in Load 1 and 2 (i.e., one side of it is colored and thus more salient). However, we are not strongly interested in this distinction and aim at using the retro-cue as the event to locate in time the onset of different demands between tasks.
}

allow time-frequency decomposition of low frequencies (Cohen, 2014b). Finally, participants were presented with the target screen, which differed depending on the task. In the Implementation task, the image of one of the cued mappings was presented centrally and participants were required to press the associated pair of keys (both index or both middle fingers). In the Memorization task, one mapping (i.e., image and associated response) was presented centrally. The image in the Memorization probe belonged to one of the selected mappings (except for catch trials, see below). Participants had to report whether the presented mapping matched one of those selected by the retro-cue, by pressing with both fingers of one hand for "yes" or both fingers of the other hand for "no". The sides for "yes" and "no" were randomly assigned on a trial basis to ensure that participants could not prepare any response during the CTI, therefore encouraging the mere declarative maintenance of the instruction memoranda. Labels with "yes" and "no" appeared at the bottom of the target screen together with the mapping. In $50 \%$ of the trials, the target screen showed the same mapping as in the encoding ("yes" response). In the other $50 \%$ of trials, one of the selected images was presented which was associated with a different response with respect to encoding ("no" response). Crucially, despite the similarities in trial structure, the Memorization task relied on the declarative maintenance of the mappings as no response could be prepared, whereas the Implementation task encouraged a proactive preparation of the selected mappings for the execution of the required action plan. Both tasks included catch trials ( $\sim 25 \%$ for each task), in which the target screen displayed a different image than the four displayed in the encoding screen. In this case, participants were required to press the spacebar. This was done to discourage participants to adopt specific strategies, such as memorizing only the mappings associated with one response option. Since we were interested in the brain activity before the onset of the target, EEG recordings from catch trials were analyzed together with regular trials. For each task, participants completed 5 experimental blocks, for a total of 180 trials (60 per load, Fig. 1).

Each of the two tasks was preceded by a practice session. This consisted of mini-blocks of 12 trials, including all possible load conditions (i.e. number of mappings selected by the retro-cue) and at least one catch trial. The only difference with the main task was the presence of feedback at the end of each trial, signaling the accuracy of the response or encouraging participants to respond faster in case no response was registered within the maximum response time of $3 \mathrm{~s}$. Performance was assessed at the end of each mini-block: if accuracy was above $80 \%$, practice was concluded, otherwise a new mini-block started, up to a maximum of 3 blocks. S-R mappings used during the practice were never presented again during the main task. The total duration of the exper- 
iment, including cap preparation, practice, main tasks and breaks was approximately $150 \mathrm{~min}$.

\subsection{EEG Recordings and pre-processing}

Electrophysiological data were recorded using a BioSemi ActiveTwo system (BioSemi, Amsterdam, Netherlands) with $64 \mathrm{Ag}-\mathrm{AgCl}$ electrodes arranged in the standard international 10-20 electrode mapping (Klem, Lüders, Jasper, \& Elger, 1999), with a posterior CMS-DRL electrode pair. Two reference electrodes were positioned at the left and right mastoids. Eye movements were registered with a pair of electrodes above and below the left eye and two additional electrodes at the outer canthi of both eyes. EEG signals were recorded at a $1024 \mathrm{~Hz}$ sampling rate.

EEG data were preprocessed using the Fieldtrip toolbox (Oostenveld, Fries, Maris, \& Schoffelen, 2010), running in MATLAB (MATLAB R2017b, The MathWorks, Inc., Natick, Massachusetts, United States). First, the data were downsampled to $512 \mathrm{~Hz}$ and re-referenced to the average of the mastoids. Then, a $0.5-45 \mathrm{~Hz}$ band-pass FIR filter was applied to the data, together with a Notch filter at $50 \mathrm{~Hz}$ and its harmonics. Data were epoched relative to the onset of the retro-cue (from -1000 to $2500 \mathrm{~ms}$ ) and demeaned to the average of the whole epoch, to improve independent component analysis (ICA) (Groppe, Makeig, \& Kutas, 2009). Only trials in which the participant performed the correct response were retained for subsequent analyses. Trials exhibiting movement artifacts or excessive noise were removed following visual inspection of the data. Next, eye movements artifacts were removed by means of ICA, using the EEGLAB (Delorme \& Makeig, 2004) runica algorithm as implemented in Fieldtrip. Components to discard were selected based on their topography, the correlation between their time course and the horizontal and vertical electrooculography, and their power spectrum. For most participants, two components were removed (capturing blinks and horizontal eye movements, respectively); in six participants blinks were reflected in two components, leading to the removal of three IC, and in two participants only one component was removed. Finally, data were visually inspected again to ensure successful cleaning and one $(\mathrm{N}=6)$, two $(\mathrm{N}=2)$ or three $(\mathrm{N}=1)$ excluded channels were interpolated by means of spherical spline interpolation (Perrin, Pernier, Bertrand, \& Echallier, 1989). This cleaning procedure resulted in an average of 151.09 trials for the Implementation task $(S D=12.29,83.94 \%)$ and 143.06 trials for the Memorization task $(S D=14.07,79.48 \%)$. For each load condition, an average of $81.7 \%$ of trials were retained (Implementation, Load 1: $52.14(S D=3.64)$ trials, Load 2: 50.26 $(S D=5.19)$ trials, Load 4: 48.68 trials $(S D=5.74)$; Memorization, Load 1: 49.88 trials $(S D=4.84)$, Load 2: 47.86 trials $(S D=5.86)$, Load 4 : 45.31 trials $(S D=5.94)$.

\subsection{Spectral Analysis}

For each Task $\mathrm{x}$ Load condition (Memorization-Load 1, Memorization-Load 2, Memorization-Load 4 and Implementation-Load 1, Implementation-Load 2, Implementation-Load 4), time-frequency analysis was performed separately using complex Morlet wavelet convolution, to estimate spectral power from 1 to $45 \mathrm{~Hz}$ in steps of $1 \mathrm{~Hz}$. The number of cycles in the wavelet was frequency-specific, ranging from 2 at $2 \mathrm{~Hz}$ and linearly spaced up to 7 cycles at $45 \mathrm{~Hz}$, to achieve a good trade-off between temporal and frequency precision (Cohen, 2014a). This analysis resulted in one time-frequency spectrogram for each channel, condition and participant. Condition-specific decibel normalization was then applied, using the time window between -500 and $-200 \mathrm{~ms}$ before the onset of the retro-cue as baseline. Direct comparison of the baseline time window between the two Tasks excluded the presence of differences induced by the blocked design (see Supplementary material). To avoid data contamination due to the smearing in time of the processing of the target, all statistical analyses are performed on the time window from 0 to $1800 \mathrm{~ms}$ with respect to retro-cue onset, therefore leaving $100 \mathrm{~ms}$ gap between the end of our analyses window and the earliest jittered target onset.

\subsection{Statistical Analyses}

Reaction times (RTs) and error rates (ER) were separately entered in 2 (Task: Memorization vs Implementation) x 3 (Load: 1, 2, 4) repeated measure ANOVAs, performed in JASP (Jasp Team, 2019).

To evaluate the statistical significance of differences between EEG time-courses or time-frequency spectra, we adopted a cluster-based permutation approach (Maris \& Oostenveld, 2007), which is appropriate to assess the reliability of neural patterns over neighboring data points. Moreover, this approach is robust against the multiple-comparison problem, as the significance of clusters found in the observed group-level data is estimated against a distribution of clusters obtained by randomly permuting the assignment of participants to each group. First, depending on the number of conditions to be compared, we performed either a two-sided t-test (two conditions) or an F-test (three conditions), at an $\alpha$ level of 0.05 , between all data points of the observed conditions. Then, we considered as cluster a group of adjacent data points with same sign significance and as cluster-size the sum of all t- or F-values in the cluster. Next, we used 5,000 permutations of participant-level data to estimate a distribution of cluster sizes under the null hypothesis that there were no differences between conditions. The $P$-value for each cluster in the observed group-level data corresponds to the proportion of permutations in which the largest cluster size was larger than the size of the considered observed cluster. Again, we used a significance alpha level of 0.05 , therefore only observed clusters whose size was larger than the size of the largest cluster in at least $95 \%$ of permutations are reported. This approach provides a statistically sound procedure to compare conditions and establish the existence of a difference between them, reliably accounting for multiple comparisons. However, because clusters found through this procedure reflect the result of the second level inference between the observed and the null permuted distributions, no strong conclusions can be drawn with respect to their boundaries, such as the exact latency or frequency range of the effect (Sassenhagen \& Draschkow, 2019).

\subsection{Contralateral alpha suppression}

Consistent findings reported alpha suppressionover posterior regions contralateral to the attended spatial location, indicating top-down anticipatory mechanisms of attentional orienting in the perceptual or internal space (Bonnefond \& Jensen, 2012; Capilla et al., 2014; Gould et al., 2011; Jensen \& Mazaheri, 2010; Mok et al., 2016; Myers et al., 2015; Rihs et al., 2007, 2009; Rohenkohl \& Nobre, 2011; Sauseng et al., 2005; Thut et al., 2006; van Dijk et al., 2008; Wallis et al., 2015).

To estimate the suppression of alpha oscillations contralateral to the cued hemifield, we averaged the time-frequency spectra corresponding to Load 1 and Load 2, separately for the two tasks. Trials with neutral retro-cues (Load 4) were excluded from this analysis because they did not induce attentional orienting towards one of the two visual hemifields. Based on previous literature, two pairs of electrodes were selected in right (P8, PO8) and left (P7, PO7) posterior parietal regions (de Vries, van Driel, \& Olivers, 2019a; Gould et al., 2011; Schneider et al., 2017; van Ede, Chekroud, Stokes, \& Nobre, 2019b). Power spectra were extracted from these electrode pairs, averaged in the alpha frequency range $(8-14 \mathrm{~Hz})$, and collapsed between retro-cues pointing to the left and right hemifield, in order to extract one power time series for the contralateral hemisphere and one for the ipsilateral hemisphere. The two time series were compared by means of clusterbased permutation testing, separately for the two tasks (see Statistical Analyses section). Additionally, the cluster-based permutation approach was also used to compare the contralateral alpha suppression between the two tasks. Namely, for each task the ipsilateral power time series 
was subtracted to the contralateral one, separately for each participant, and the resulting difference waves were compared. This analysis was performed to test whether the deployment of alpha oscillations to orient attention towards relevant mental representations differed between tasks.

\subsection{Load-dependent alpha increase}

Posterior alpha power has been observed to increase with the number of items relevant after attentional selection in a WM task (Fukuda, Mance, \& Vogel, 2015; Jensen, 2002), also following retrospective selection (Manza et al., 2014; Poch et al., 2017, 2018). Observing such modulation in our data would be evidence in favor of an effective selection of the relevant mappings. To investigate the increase in alpha power relative to the number of retained items, we extracted the average power for the frequencies ranging from 8 to $14 \mathrm{~Hz}$, separately for each condition and task, from the electrode pair $\mathrm{Pz}$ and $\mathrm{POz}$ (Jensen, 2002). This operation led to one averaged alpha power timecourse for each Task-Load combination per participant. We then used the cluster-based approach described in the Statistical Analyses section to test for the main effects of Task, Load, and their interaction.

\subsection{Mid-frontal theta in Implementation}

The two tasks differ to the extent they require and allow the reformatting of the encoded information in an action-oriented code. We hypothesized such transformation to rely on a top-down mechanism supported by low frequency oscillations. To test our predictions, timefrequency spectra were extracted from the CTI $(0-1800 \mathrm{~ms})$ in the frequency range 3-7 Hz, averaged from channels Fz and AFz. The choice of these two channels was guided by both their widespread use in the literature concerning midfrontal theta (Berger et al., 2019; Cooper et al., 2019; Onton et al., 2005; Popov et al., 2018; Senoussi et al., 2020) and a condition-independent channel selection procedure. Namely, we fitted the power spectra from all channels, computed across all trials, regardless of the Load condition and the Task, with the FOOOF toolbox (Haller et al., 2018). This method allows to decompose different components of the power spectrum (i.e., the aperiodic ( $1 / \mathrm{f}$ pattern) and period components) and thus to better capture the oscillatory profile of electrophysiological data. This analysis confirmed that, across conditions, channels Fz and AFz exhibited the largest peaks in the power spectra between 3 and $7 \mathrm{~Hz}$ (Supplementary material, Figure S3). No differences between Tasks or Load conditions were observed in the parameters of the aperiodic component.

We tested the main effect of Task by comparing the time-frequency spectra of Implementation and Memorization (averaged across levels of Loads) by means of cluster-based permutation test. Similarly, we tested the main effect of Load by contrasting the spectra for each level of Load, averaged across Task condition. We then tested for a significant effect of the interaction between our two factors by running a cluster-based permutation test on the three time-frequency spectra resulting from pairwise subtraction of the two tasks for each Load level (i.e., Implementation 1 minus Memorization 1, Implementation 2 minus Memorization 2 and Implementation 4 minus Memorization 4).

\subsection{Motor-related $\mathrm{Mu}$ and beta suppression in Implementation}

Analogously, we hypothesized that action-oriented procedural representations should entail a motor plan, reflected in stronger signatures of proactive motor preparation. We expected differences in two frequency bands associated with motor preparation, namely $\mathrm{mu}(8-12 \mathrm{~Hz})$ and beta (15-30 Hz) (Cheyne, 2013; Pineda, 2005). To test for this, we extracted time-frequency spectra averaged across electrodes C3 and C4 from the CTI (0-1800 ms). Next, we again used the cluster-based permutation approach outlined for mid-frontal theta, to look for significant clusters of different activity between tasks, loads, and their interaction.
We selected electrodes C3 and C4 because we expected the effect to be maximal over motor and pre-motor cortices ${ }^{2}$ (Marchesotti, Bassolino, Serino, Bleuler, \& Blanke, 2016; McFarland, Miner, Vaughan, \& Wolpaw, 2000). Moreover, we averaged across the two of them because the goal of this comparison was to investigate pre-movement preparatory oscillatory activity, rather than the effector-specific M1 activation (Neuper \& Pfurtscheller, 2001; Pfurtscheller, Brunner, Schlögl, \& Lopes da Silva, 2006; Pfurtscheller \& Neuper, 1997). In our study, responses were bimanual in the Implementation task and lateralized in the Memorization task. However, we reasoned that our test is independent of such difference, as before target onset responses from both hands had to be prepared in the two tasks.

\section{Results}

\subsection{Behavioral results}

Concerning behavioral performance, we expected valid retro-cues to have a beneficial effect on reaction times and error rates in both tasks (Souza \& Oberauer, 2016). Repeated measures ANOVA on RTs confirmed a significant main effect of $\operatorname{Load}^{3}\left(F_{1.44,49.06}=104.65, p<0.001\right.$, $\eta_{\mathrm{p}}^{2}=0.76$ ): RTs increased with the number of mappings selected by the retro-cue (Load 1: $M=978 \mathrm{~ms}, S D=196$, Load $2: M=1168 \mathrm{~ms}$, $S D=150$, Load 4: $M=1266 \mathrm{~ms}, S D=142)$. The main effect of Task was also significant $\left(F_{1,34}=237.20, p<0.001, \eta^{2}{ }_{p}=0.87\right)$, with slower RTs in Memorization $(M=1355 \mathrm{~ms}, S D=185)$ than Implementation $(M=907 \mathrm{~ms}, S D=160)$. The interaction between the two factors also resulted to be significant $\left(F_{1.32,44.88}=4.42, p=0.031, \eta^{2}{ }_{p}=0.11\right)$. More specifically, the effect of Load was larger in the Implementation task $\left(F_{1.44,49.06}=93.20, p<0.001\right)$ compared to the Memorization task $\left(F_{1.44,49.06}=45.50, p<0.001\right)$ (Fig. 2).

Error rates showed a significant main effect of Task $\left(F_{1,34}=11.08\right.$, $\left.p=0.002, \eta^{2}{ }_{p}=0.25\right)$ and of Load $\left(F_{1.67,57.91}=25.65, p<0.001\right.$, $\left.\eta^{2}{ }_{\mathrm{p}}=0.43\right)$. Participants were significantly more accurate in the Implementation task $(M=0.14, S D=0.09)$ compared to the Memorization task $(M=0.19, S D=0.10)$, and were more accurate when less items were selected by the retro-cue (Load 1: $M=0.12, S D=0.07$, Load 2: $M=0.17, S D=0.10$, Load 4: $M=0.20, S D=0.12$ ). The interaction between the two factors was not significant ( $p=0.266$ ) (Fig. 2).

Regarding catch trials, participants could successfully detect a new image in both the Memorization task (error rate in catch trials: $M=0.13$, $S D=0.08)$ and the Implementation task $(M=0.09, S D=0.07) . \mathrm{Nev}-$ ertheless, they were significantly less accurate $\left(t_{34}=3.16, p=0.003\right.$, $d=0.53$ ) in the Memorization task.

Such differences in performance between Implementation and Memorization are not surprising and can be at least partially attributed to the task-specific processes taking place after the occurrence of the target (Formica et al., 2020; Muhle-Karbe et al., 2017). Specifically, the Memorization task requires the comparison of the presented mapping to the encoded ones, the identification of the response side, and the execution of the intended response. On the contrary, the Implementation task only involves the identification of the target stimulus and the execution of the associated motor response.

\footnotetext{
2 Electrodes selection for analyses in the mu and beta frequency ranges was based on the literature and on the clearer assumptions on the brain areas causing the expected oscillations (i.e., pre-SMA and M1). Moreover, we chose not to rely on an independent electrode selection as we did for theta because the FOOOF toolbox results in higher frequencies are more susceptible to muscular artifacts and therefore less accurate.

3 Mauchly's test revealed that the assumption of sphericity is violated ( $\mathrm{p}<$ 0.05). Greenhouse-Geisser correction is applied here and in all results where the sphericity assumption is violated.
} 

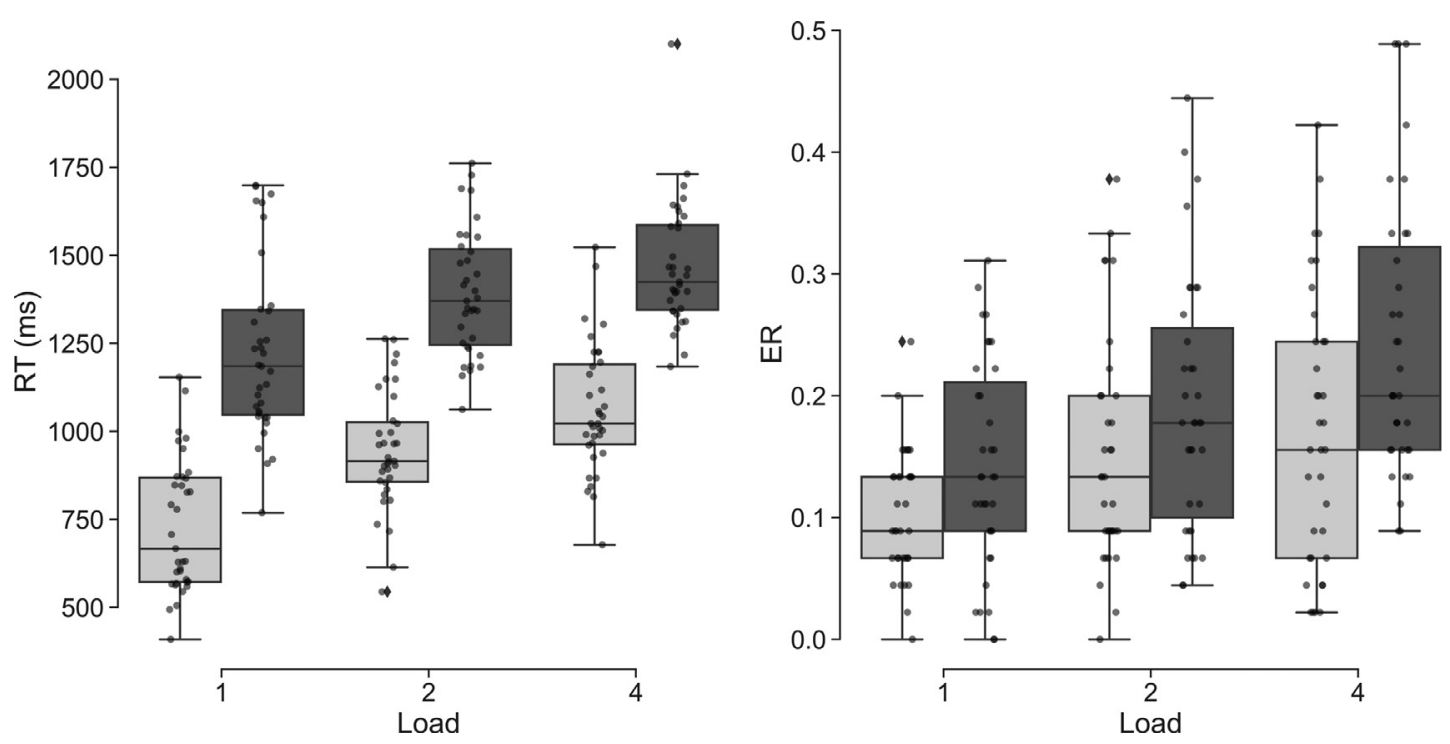

Task Implementation Memorization

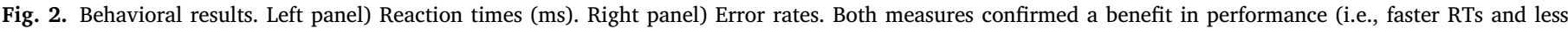

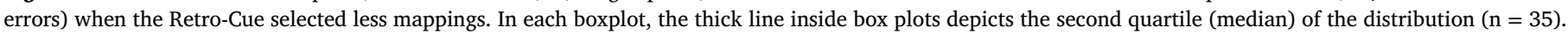

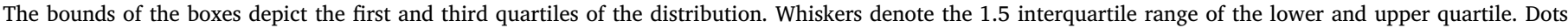
represent individual subjects' scores.

\subsection{EEG Results}

\subsubsection{Contralateral alpha power decrease}

As hypothesized, we observed a stronger reduction in alpha power in contralateral compared to ipsilateral electrodes across loads 1 and 2 both in Implementation ( $p=0.013$, cluster-corrected) and Memorization ( $p=0.003$, cluster-corrected) tasks (see Supplementary material for the same analysis performed separately for the two load conditions, Figure S2). The significant clusters spanned the time window around 600$800 \mathrm{~ms}$. Notably, we did not find any difference between the two tasks (no clusters were found in the observed data), supporting our hypothesis that the mechanisms allowing for attentional orienting are engaged to a similar extent in the two tasks (Fig. 3a). To qualitatively confirm our electrodes selection and the location of the significant effect, contralateral minus ipsilateral half-topographies are reported separately for each task (Fig. 3b).

\subsubsection{Load-dependent alpha increase}

We predicted alpha power to also track the number of items selected by the retro-cue to be relevant for the ongoing task. As expected, we found significant differences in the time courses of posterior alpha power between different Loads ( $p<0.001$, cluster corrected). The cluster spanned from $500 \mathrm{~ms}$ after the onset of the Retro-Cue to the end of the CTI (Fig. 3c). Pairwise comparisons confirmed that Load 2 had significantly larger alpha power compared to Load $1\left(p_{1}=0.001, p_{2}=0.002\right)$, and lower with respect to Load $4(p<0.001)$. Scalp topographies of alpha power in the time window $500-1800 \mathrm{~ms}$ are reported separately for each Task and Load condition (Fig. 3d). Crucially, no cluster was found when testing for the main effect of Task, and the larger cluster yielded by the interaction of the two factors did not survive correction for multiple comparisons $(p=0.10)$.

\subsubsection{Mid-frontal theta in Implementation}

We expected to find stronger theta power over mid-frontal electrodes in the Implementation task compared to the Memorization task. Supporting this prediction, we found a significant cluster ( $p=0.04$, corrected for multiple comparisons) when comparing the power estimates of the two tasks in the frequency range 3-7 Hz from electrodes $\mathrm{Fz}$ and AFz (Fig. 4). On the contrary, the test aimed at detecting a main effect of Load approached but did not reach significance $(p=0.09)$. Plots depicting theta oscillations separately for each condition can be found in the Supplementary material (Figure S6).

Moreover, our corollary hypothesis concerning theta dynamics predicted such effect to be modulated by the number of mappings to be implemented or memorized. Specifically, we expected that in Load 4 Implementation and Memorization would not differ, in line with behavioral findings showing that 4 novel instructions cannot be proceduralized at once and would therefore be maintained in a declarative format in both conditions (Liefooghe et al., 2012). However, the effect of the interaction resulted to be not significant $(p=0.53)$. Despite the lack of a significant interaction, and thus the impossibility to draw any strong conclusion from post-hoc tests, we explored the effect of Task across different Load levels by means of pairwise comparisons. We observed a significant cluster only in the contrast Implementation 2 vs Memorization $2(p=0.02)$, whereas no cluster was observed for Load 4 , and no cluster survived correction in Load $1(p=0.23)$.

One potential confound when investigating activity in low frequency bands is that this might be reflecting sustained ERPs. To rule out this possibility, we run two additional analysis checks. First, we compared across tasks the Contingent Negative Variation (CNV), a slow negative potential over centro-frontal electrodes during preparation for an upcoming target (Walter, Cooper, Aldridge, McCallum, \& Winter, 1964). The CNV did not show a differential pattern between Implementation and Memorization (Supplementary material, Figure S4). Moreover, to additional discard the alternative explanation of a sustained ERP, we repeated our contrast performing the time-frequency decomposition after averaging the Implementation and the Memorization trials, separately. In this way, activity phase-locked to the onset of the retro-cue gets averaged out, and differences in the resulting time-frequency spectra would be reflecting the evoked response. Again, we found no differences between tasks (Supplementary material, Figure S5). Taken together, these analyses suggest that our frontal activity is better described by low frequency oscillations, rather than a sustained ERP. Furthermore, at the lowest estimated frequency of $2 \mathrm{~Hz}$, the wavelet was two-cycles long, i.e. spanning a $1 \mathrm{~s}$ interval, which is considered to be selective to true oscillations and able to disentangle them from non-oscillatory signal (Cohen, 2014a). Finally, interpreting this frontal activity as the correlate of target expectancy would not account for the effect specific to Load 2 observed in time-frequency analyses. 
a

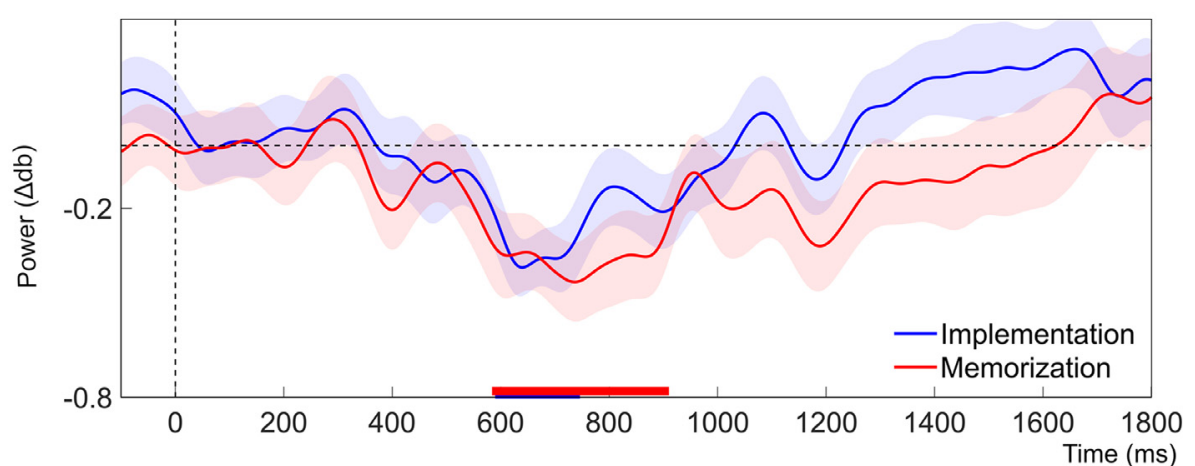

b
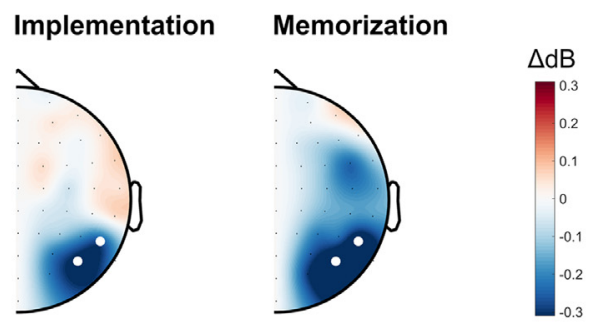

$8-14 \mathrm{~Hz}$

$600-800 \mathrm{~ms}$
Fig. 3. Alpha power dynamics. a) Time courses of the difference waves (contralateral minus ipsilateral time course) of alpha power activity between contralateral and ipsilateral electrode clusters (PO8 - P8 and PO7 - P7). Both difference waves show a significant deflection from 600 to $800 \mathrm{~ms}$ after the onset of the retro-cue. Horizontal lines above the $\mathrm{x}$-axis indicate significant temporal clusters (blue: Implementation, red: Memorization), obtained with cluster-based permutation testing). Shading represents the s.e.m., calculated across participants $(n=35)$. b) Half topographies showing differences in alpha power for contralateral minus ipsilateral electrodes collapsed across hemispheres. White dots indicate the a-priori selected electrodes used in the cluster-based permutation analysis. c) Alpha power time courses from electrodes $\mathrm{Pz}$ and POz, averaged across tasks. The thick horizontal line above the $\mathrm{x}$-axis indicates a cluster of significant difference between the Load conditions, obtained with cluster-based permutation testing. Shading represents the s.e.m. calculated across participants $(n=35)$. d) Topographies of each Task $x$ Load condition. White dots indicate the a-priori selected electrodes ( $\mathrm{Pz}$ and $\mathrm{POz})$.
C

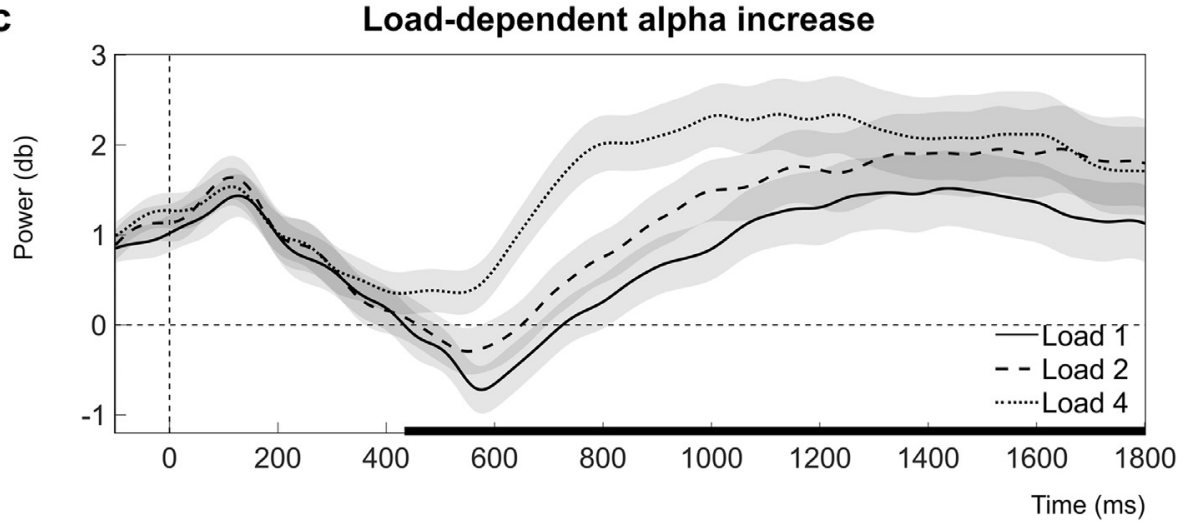

d

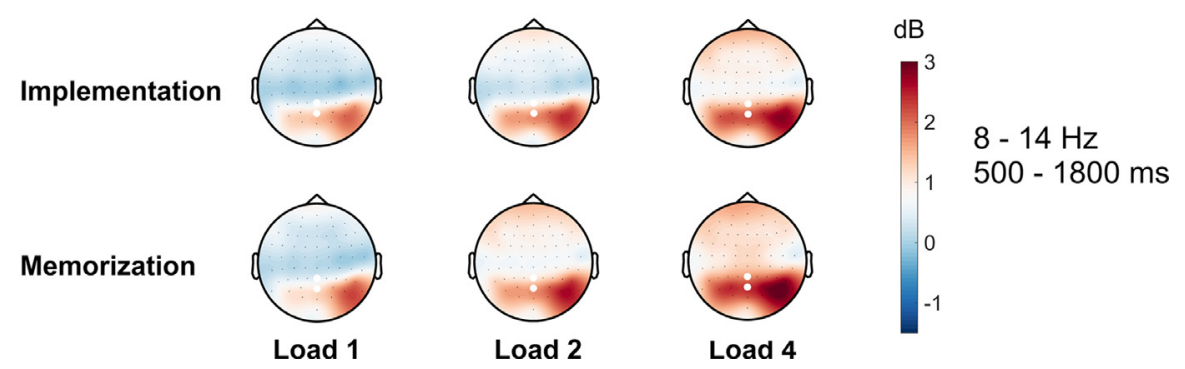

\subsubsection{Motor-related mu and beta suppression in Implementation}

We hypothesized that proceduralization would induce increased recruitment of motor and premotor regions, in preparation for the instructed upcoming movement, compared to Memorization. Specifically, we predicted a suppression of oscillations in the $\mathrm{mu}(8-12 \mathrm{~Hz})$ and beta $(15-30 \mathrm{~Hz})$ frequency ranges, which have been shown to reflect motor cortex activity (Cheyne, 2013; Pineda, 2005; Rhodes et al., 2018; Schneider et al., 2017; Tzagarakis et al., 2015). To test for this hypothesis, we again used cluster-based permutations and we tested the effects of Task, Load, and their interaction, separately for each frequency range of interest (i.e. mu and beta).
In line with our prediction, we found significant clusters when contrasting the two Tasks both in the mu $(p=0.01)$ and beta $(p=0.03)$ frequency ranges. These findings support the idea that proceduralization involves a recruitment of motor areas for the activation of the instructed motor plan (Fig. 5).

Similarly, the effect of Load resulted to be significant in both mu $(p<0.001)$ and beta $(p<0.001)$ range, suggesting that these motorrelated oscillatory features are also sensitive to the number of items being handled. Plots depicting mu and beta oscillations separately for each condition can be found in the Supplementary material (Figure S7). 
a

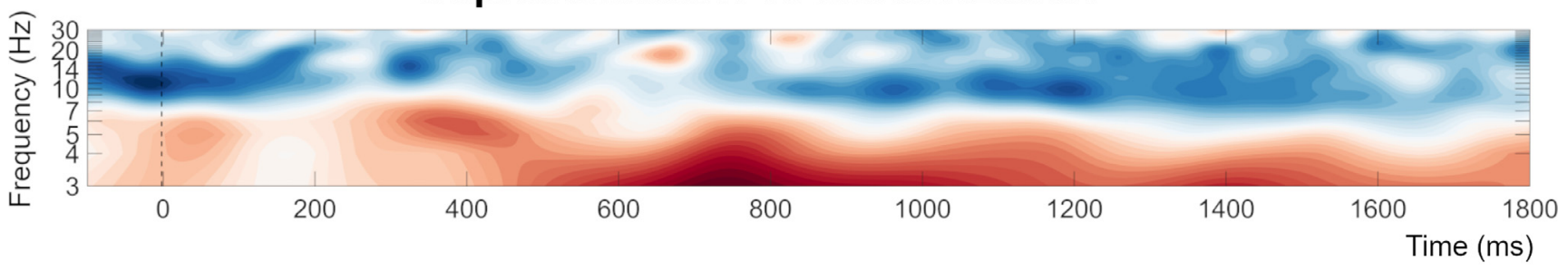

b

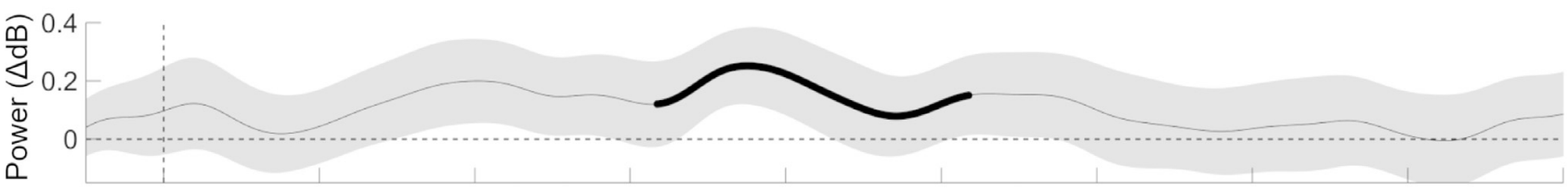

C

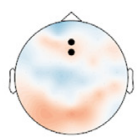

0-200

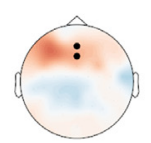

200-400

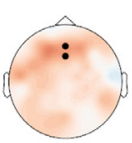

400-600

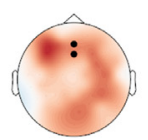

$600-800$

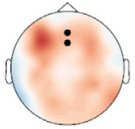

800-1000

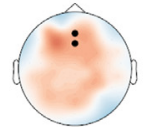

1000-1200

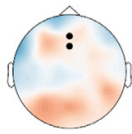

1200-1400

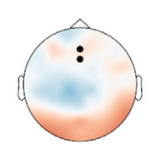

1400-1600

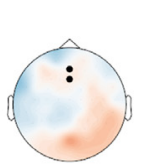

1600-1800 ms

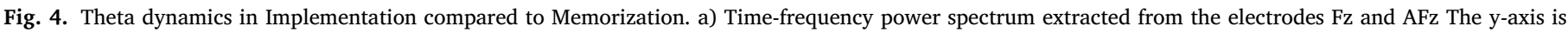

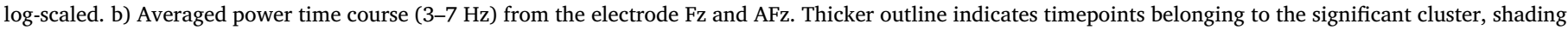

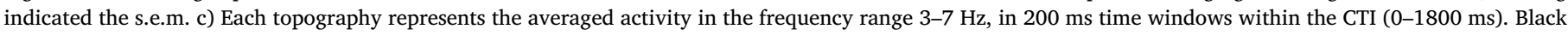
dots indicate the electrodes used for the statistical analysis.

As for theta, our secondary hypothesis was that the differences in mu and beta dynamics between tasks would be modulated by the number of mappings. This hypothesis would be in line with a capacity limit in the amount of instructions that can be proceduralized at once, and thus converted in an action plan. However, in neither of the two frequency bands the cluster-based permutations tests revealed significant clusters for an effect of such interaction (mu: $p=0.14$, beta: $p=0.57$ ). As exploratory follow-up analyses we performed pairwise comparisons separately for each Load level (i.e., Implementation 1 vs Memorization 1, Implementation 2 vs Memorization 2, Implementation 4 vs Memorization 4). Although interpretations of these results have to be cautious and do not allow to draw any strong statistical conclusion, it is interesting to observe that again only in Load 2 the two tasks showed clusters of different activity (beta: $p_{1}<0.001, p_{2}=0.02, p_{3}=0.21$, mu: $p_{1}=0.003$, $p_{2}=0.006, p_{3}=0.042$ ). On the contrary, comparisons in Load 1 (beta: $p=0.19$, mu: no cluster) and Load 4 (beta: $p=0.35$, mu: $p=0.13$ ) yielded no significant clusters.

\section{Discussion}

In the present study, we investigated the spectral and temporal dynamics underlying novel instruction implementation. In line with our predictions and the framework proposed by Myers and colleagues (2017), we observed an analogous unfolding of early attentional mechanisms across tasks, reflected in similar patterns of alpha power dynamics. Crucially, Implementation and Memorization also showed differences in frontal theta, and central mu and beta oscillations, suggesting that Implementation-specific processes go beyond the attentional prioritization of the relevant items. These mechanisms are likely involved in the reformatting of the selected S-R mappings into a behavior-optimized, action-bound process requiring the exertion of cognitive control and resulting in the preparation of the instructed motor plan (van Ede, 2020).

Importantly, although our two tasks differ in terms of behavioral performance, it is unlikely that the observed neural differences reflect more general distinctions between task conditions. If that was the case, one would expect the task characterized by higher demands and lower performance (i.e., Memorization) to be associated with neural features of cognitive effort, such as higher frontal theta, which has been shown to increase with cognitive load (Onton et al., 2005). In our data we observed the opposite pattern, increasing our confidence that the neural oscillations reflect the hypothesized cognitive processes rather than a more general signature of task difficulty or engagement.

An underlying assumption of our study is that the intention to implement the instructed mappings is a crucial necessary factor to trigger the reformatting from declarative to procedural state (Liefooghe et al., 2012). This exclusive role of the intention to implement has been questioned by some recent behavioral evidence, suggesting an automatic reformatting of the instruction independent of task demands (Liefooghe \& De Houwer, 2017). This might be due to the specific response set used in the specific task, or to other idiosyncratic factors. Despite these inconsistencies in the behavioral measures, the dissociation between Implementation and Memorization is well established at the neural level (Demanet et al., 2016; González-García et al., 2019; Muhle-Karbe et al., 2017). Here, we show such differences are reflected in oscillatory mechanisms.

\subsection{Alpha-mediated attentional orienting is analogous in Implementation and Memorization}

Activity in the alpha frequency band has been consistently associated with attentional processing (van Ede, 2017). In particular, posterior alpha power decreases over posterior electrodes contralateral to the attended hemifield, both in perceptual and in WM tasks (Jensen \& Mazaheri, 2010; Poch et al., 2017; Sauseng et al., 2005). In our experiment, participants were encouraged to focus on the selected items and discard the unselected ones. Coherently, in both tasks we found after the retrocue a significant suppression of alpha oscillations contralateral to the attended hemifield. The most influential model proposed to account for this phenomenon is referred to as Gating by Inhibition (Jensen \& Mazaheri, 2010). According to this framework, top-down alpha modulation of sensory cortices allows for the inhibition of irrelevant inputs, contributing to the creation of a functional network optimized to perform the task (Mazaheri et al., 2014; Van Diepen, Foxe, \& Mazaheri, 2019). Our clusters of alpha lateralization extended around $600-800 \mathrm{~ms}$ after retro-cue. This dynamic is consistent with previous findings on lateralized retrospective orienting of internal attention, showing modulations 
a Implementation vs Memorization

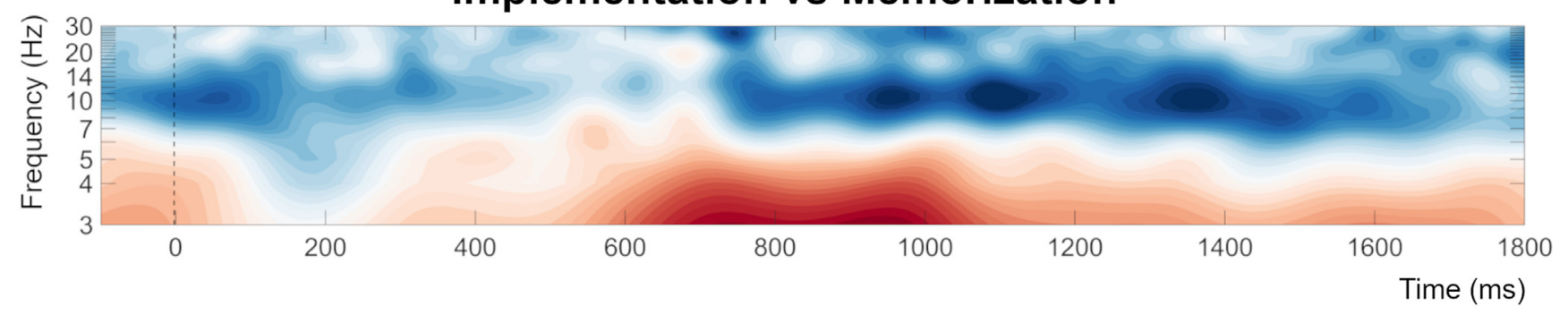

b

$\mathrm{Mu}$

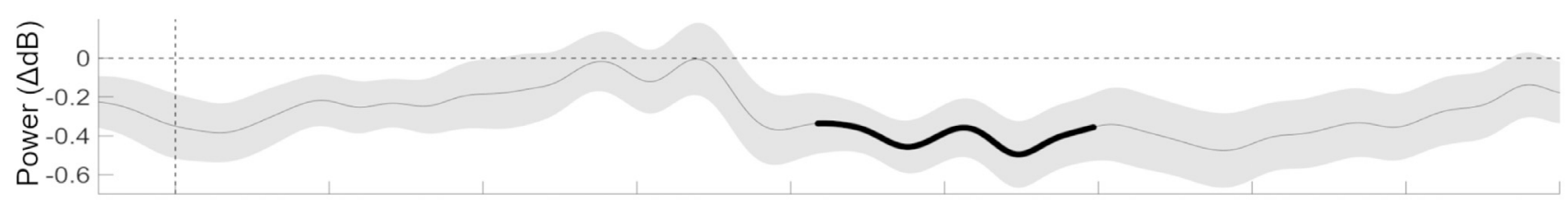

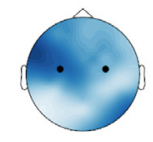

$0-200$

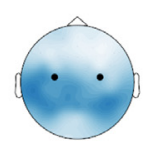

$200-400$

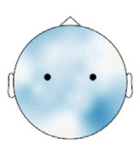

$400-600$

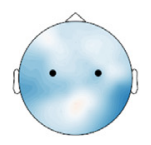

$600-800$

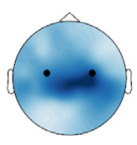

$800-1000$

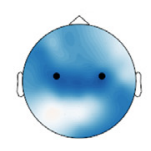

$1000-1200$
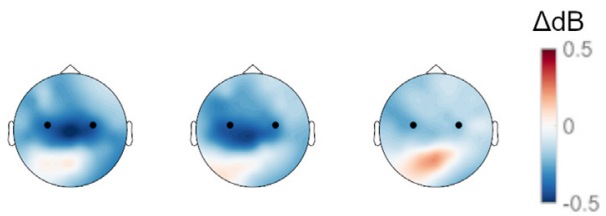

$1200-1400$

$1400-1600 \quad 1600-1800 \mathrm{~ms}$

C Beta

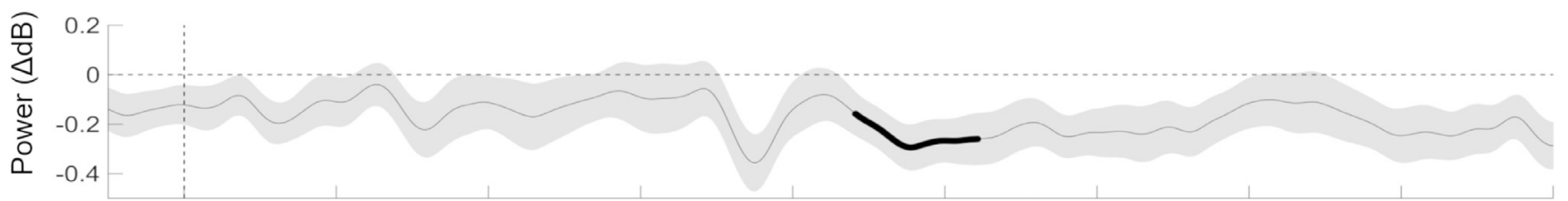

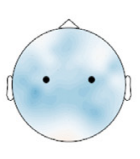

$0-200$

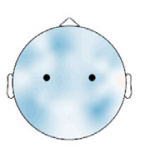

$200-400$

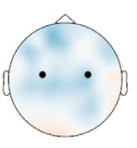

400-600

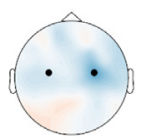

$600-800$

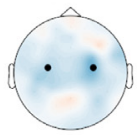

800-1000

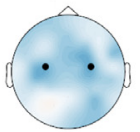

$1000-1200$

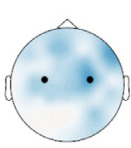

$1200-1400$

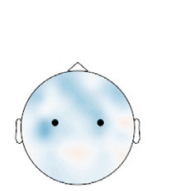

$1400-1600 \quad 1600-1800 \mathrm{~ms}$

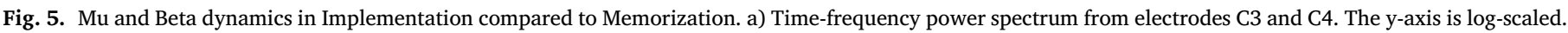

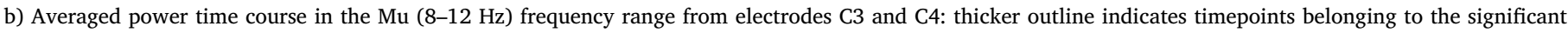

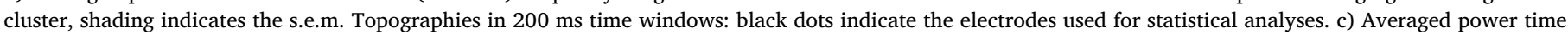

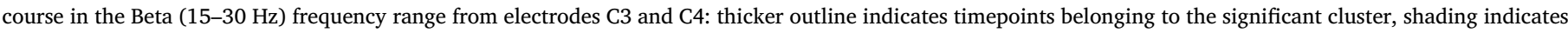
the s.e.m. Topographies in $200 \mathrm{~ms}$ time windows: black dots indicate the electrodes used for statistical analyses.

within 500 and 1000 ms (Mok et al., 2016; Poch et al., 2017, 2018; Wallis et al., 2015; Wolff, Ding, Myers, \& Stokes, 2015; Wolff, Jochim, Akyürek, \& Stokes, 2017). Moreover, it has been shown recently that endogenous orienting of attention elicits lateralized alpha activity with onset around $600 \mathrm{~ms}$, later with respect to exogenous cues $(\sim 250 \mathrm{~ms})$ (Keefe \& Störmer, 2021).

The occurrence of the selection process is further supported by loaddependent centro-posterior alpha power modulation. The amplitude of alpha power has been observed to be sensitive to the number of items retained during a WM task (Fukuda et al., 2015; Jensen, 2002). In both our tasks, alpha increased with load. Crucially, we observed no taskdependent differences in the dynamics of alpha oscillations, suggesting that the information provided by the retro-cue is used to analogously orient attention and select the items that are likely to be probed, independently of task demands.

\subsection{Low frequencies oscillations for $S-R$ binding in Implementation}

We predicted low frequency oscillations over frontal sensors to be associated with the proactive reformatting of the S-R mapping(s) into an action-oriented code. The crucial contribution of the PFC in quickly converting symbolic instructions into task-sets for prospective action has been widely established in several fMRI studies (Brass, Wenke, Spengler, \& Waszak, 2009; Cole, Bagic, Kass, \& Schneider, 2010; Dumontheil, Thompson, \& Duncan, 2011; González-García et al., 2017; Hartstra et al., 2011; Palenciano, González-García, Arco, \& Ruz, 2019; 
Ruge \& Wolfensteller, 2010). In particular, declarative maintenance and implementation of novel mappings can be dissociated using univariate and multivariate analyses in prefrontal regions, such as the inferior frontal junction (IFJ) and the inferior frontal sulcus (IFS) (Bourguignon et al., 2018; Demanet et al., 2016; Hartstra et al., 2011; Muhle-Karbe et al., 2017). Activation of these areas has been interpreted as reflecting the creation and the activation of procedural conditionaction rules from the instructed mappings, a process that is thought to involve the manipulation of the available information and the exertion of cognitive control (Brass et al., 2017). Analogously, the need for top-down control over complex goal-directed cognitive operations is traditionally linked with oscillations in the theta frequency range over prefrontal cortices (Cavanagh \& Frank, 2014; Cooper et al., 2019). Moreover, recent influential models of large-scale brain interactions attribute to theta the crucial role of orchestrating information exchange between distant areas by synchronizing their firing pattern (Fries, 2005, 2015; Lisman \& Jensen, 2013; McLelland \& VanRullen, 2016; Verguts, 2017). We hypothesized the Implementation task in our experiment to require a more extensive allocation of cognitive control, given the need to manipulate the declarative representations of the selected S-R mappings into their procedural counterparts, leading to increased power in low frequencies over frontal sensors. Supporting this prediction, our Implementation and Memorization tasks differed in the extent to which they engaged oscillations in the theta frequency range over prefrontal regions.

Previous studies show mixed findings with respect to the role of midfrontal theta power in dissociating maintenance and manipulation of WM items. Berger and colleagues (2019) reported no differences in theta power between retention and mental spatial mirroring (i.e., manipulation) of a grid of colored dots. On the contrary, the study by Itthipuripat and colleagues (2013) used a sequence updating paradigm and revealed increased theta for both contrasts Manipulation vs Maintenance and Manipulation (correct trials) vs Manipulation (incorrect trials). Interestingly, they found low theta $(2-4 \mathrm{~Hz})$ to be sensitive to manipulation demands, whereas high theta $(5-7 \mathrm{~Hz})$ increased in both contrasts (Itthipuripat et al., 2013). Although the exact boundaries of our significant cluster cannot be strongly interpreted due to the nature of the statistical approach we adopted (see Statistical Analyses section in the Methods), the effect of Task we find in our study appears to be predominant in the lower frequencies. One possibility is that different frequencies within the theta band serve different cognitive functions. This view has recently been gaining support. Evidence in favor of a more fine-grained characterization of theta oscillations include variations in theta peak frequency depending on task demands and difficulty (Senoussi et al., 2020), and differences in the functional significance of high (posterior) and low (anterior) hippocampal theta (Goyal et al., 2020).

A functional role has been attributed to theta oscillations in the prioritization of relevant information in visual WM. Oscillations in the delta/low theta $(2-6 \mathrm{~Hz})$ range have been observed during switches of internal attention from one search template to another for prospective use (de Vries et al., 2018; de Vries, van Driel, \& Olivers, 2019b; Senoussi et al., 2019). Such involvement has also been causally tested by applying rhythmic $(5 \mathrm{~Hz})$ and arrhythmic transcranial magnetic stimulation to the lateral PFC during a change detection task with retrocues (Riddle et al., 2020). Rhythmic stimulation caused an entrainment of the ongoing theta oscillations and benefitted performance, compared with the arrhythmic train of pulses (Lakatos, Gross, \& Thut, 2019; Riddle et al., 2020; Thut, Schyns, \& Gross, 2011; Thut, Veniero, et al., 2011).

In our study, the difference in theta activity observed between tasks suggests that its function might go beyond the exertion of short-lasting top-down control over attentional prioritization, but also encompasses the reformatting of the prioritized memoranda into behavior-guiding representations. This is possible for the Implementation task, whereas in the Memorization task the prioritized representations can only be used as a template to guide the upcoming task (de Vries, Slagter, \& Olivers, 2019; Olivers \& Eimer, 2011; Olivers, Peters, Houtkamp, \& Roelfsema, 2011).

Therefore, the stronger theta activity in Implementation likely reflects the inherently more extensive reformatting process of proceduralization, which we assume involves a theta-driven binding between the stimulus and its corresponding motor plan (Combrisson et al., 2017; Engel, Roelfsema, Fries, Brecht, \& Singer, 1997; Verguts, 2017). The Memorization task also requires the prioritization and the binding of stimulus and response, but this would engage theta dynamics to a lesser extent, given the impossibility to activate a motor plan.

Although strong conclusions cannot be made with respect to timing due to the temporal smearing inherent to time-frequency analyses, attentional orienting and theta-mediated binding seem to be occurring simultaneously. The temporal extents of the two clusters are, in fact, largely overlapping. Therefore, our results hint at a parallel engagement of the processes that underlie these frequencies.

\subsection{Motor preparation in Implementation is reflected in $\mathrm{Mu}$ and beta suppression}

Previous fMRI studies showed that the proactive proceduralization of novel instructions is accompanied by increased neural activity in pre-motor areas, suggesting motor preparation (Bourguignon et al., 2018; Hartstra et al., 2011, 2012; Muhle-Karbe et al., 2017; Ruge \& Wolfensteller, 2010). In current views, the recruitment of motor areas is thought to boost instruction implementation by enabling mental simulation of the overt application of the instructed mappings (Brass et al., 2017). Motor imagery has been associated with suppression in mu and beta bands over motor cortices (Cheyne, 2013; McFarland et al., 2000; Pfurtscheller et al., 2006; Pfurtscheller \& Neuper, 1997; Pineda, 2005), which we therefore predicted to occur while the participant is preparing to implement a specific motor plan, as opposed to the declarative maintenance of the mapping. Moreover, the engagement of motor areas during implementation is consistent with the creation of a functional network optimized for the rapid and efficient execution of the mappings.

In line with our hypothesis, we found these markers of motor preparation to be higher in Implementation compared to Memorization, already during the CTI. While in both tasks participants had to perform an overt motor response at the end of the trial, only during Implementation they could proactively start preparing their response to the upcoming target. This rules out the possibility that the observed differences reflect more general mechanisms of unspecific motor energization, temporal estimation or target expectancy (Nobre \& Van Ede, 2018; Van Elswijk, Kleine, Overeem, \& Stegeman, 2007; Wiener, Parikh, Krakow, \& Coslett, 2018). Overall, our results suggest that preparing to execute novel mappings involves the activation of a specific motor plan corresponding to the instructed response, to a much larger extent then the motor engagement observed in Memorization.

Concerning the timing of the implementation-induced motor engagement, the temporal extent of the significant cluster suggests a later onset with respect to alpha and theta dynamics. Although strong conclusions cannot be drawn, based on this rough interpretation of our permutation results and theoretical models such as Myers' (2017), we are inclined to speculate that motor preparation is initiated following attentional selection of the relevant mappings. From this perspective, theta would act as an overarching mechanism to bind and control the task-optimized representations. Future studies should investigate the exact timing properties and the interplay between these processes.

\subsection{Capacity limits of proceduralization}

As a corollary hypothesis, we predicted that the capacity limits of proceduralization observed in behavioral studies (Liefooghe et al., 2012) would be reflected in stronger proceduralization-related oscillatory features with fewer mappings. However, we did not observe a significant 
interaction of Task and Load in none of our frequency bands of interest. It is possible that our design was not powered enough to detect such effects. Despite the not significant interaction, for exploratory purposes we looked at the effect of Task separately for each Load. Interestingly, we observed task-related differences only in Load 2. The interpretation of these results requires caution and can only be speculative given the exploratory nature of these analyses. Yet, they could reflect that relevant oscillations become crucial when in need to coordinate multiple bindings, but still within capacity limitations. From this perspective, Load 4 would show no differences because too many S-R mappings cannot be simultaneously recoded into action-oriented representations (Liefooghe et al., 2012), and Load 1 would instead allow a more ballistic implementation of the prepared motor plan, without the need to carefully coordinate different competing procedural representations, as is the case in Load 2 . These exploratory results pave the way for future research investigating more accurately the capacity limits of proceduralization: how they are overcome with extensive practice and how they are reflected in power modulations.

\section{Conclusions}

Our study shed light for the first time on the oscillatory dynamics associated with the retrospective prioritization and proceduralization of novel instructions. We showed that alpha-mediated mechanisms of attentional orienting are in place to prioritize the relevant items, independently from the upcoming task demands. Conversely, other neural features were sensitive to the task. The implementation of novel mappings, as opposed to their declarative maintenance, is characterized by increased power in low frequencies and by stronger suppression of mu and beta activity. The former is consistent with the purported role of theta oscillations in cognitive control for the successful binding of stimulus and response into a behavior-optimized procedure, and the latter to reflect motor preparation of the instructed motor responses. Overall, our results support the idea that under optimal task conditions, proceduralization can occur proactively in preparation for the upcoming response execution demands.

Future research should investigate how proceduralization affects the underlying neural representation of the instructed mapping, under the assumption that it is recoded from a declarative to a procedural format, and how prefrontal regions mediate this reformatting process. Moreover, future studies should explore whether capacity limits of proceduralization (Liefooghe et al., 2012) are caused by concurrent activation of interfering motor plans or intrinsic to the neural mechanisms supporting the recoding of instructions into procedures, such as the theta-mediated binding.

\section{Author contribution statement}

All authors contributed to the design of the study. S.F. collected and analyzed the data, which were then interpreted together with the other authors. S.F. wrote the manuscript and all authors were involved in the revisions. M.B supervised this work.

\section{Declaration of Competing Interest}

None.

\section{Acknowledgements}

S.F. and C.G.G. were supported by Special Research Fund of Ghent University BOF.GOA.2017.0002.03. C.G.G. was additionally supported by the European Union's Horizon 2020 research and innovation programme under the Marie Sklodowska-Curie grant agreement no. 835767. M.S. was supported by Research Foundation Flanders (FWO) grant number G012816N. MB is funded by an Einstein Strategic Professorship of the Einstein Foundation Berlin.

\section{Data and code availability}

Raw data, preprocessed data and code to reproduce the results will be made available upon reasonable request.

\section{Supplementary materials}

Supplementary material associated with this article can be found, in the online version, at doi:10.1016/j.neuroimage.2021.117870.

\section{References}

Berger, B., Griesmayr, B., Minarik, T., Biel, A.L., Pinal, D., Sterr, A., Sauseng, P., 2019. Dynamic regulation of interregional cortical communication by slow brain oscillations during working memory. Nat. Commun. 10 (1), 1-11. doi:10.1038/s41467-019-12057-0.

Bhandari, A., Duncan, J., 2014. Goal neglect and knowledge chunking in the construction of novel behaviour. Cognition 130 (1), 11-30. doi:10.1016/j.cognition.2013.08.013.

Bonnefond, M., Jensen, O., 2012. Alpha oscillations serve to protect working memory maintenance against anticipated distracters. Curr. Biol. 22 (20), 1969-1974. doi:10.1016/j.cub.2012.08.029.

Bourguignon, N.J., Braem, S., Hartstra, E., De Houwer, J., Brass, M., 2018. Encoding of novel verbal instructions for prospective action in the lateral prefrontal cortex: evidence from univariate and multivariate functional magnetic resonance imaging analysis. J. Cogn. Neurosci. 26 (3), 1-15. doi:10.1162/jocn_a_01270.

Brady, T.F., Konkle, T., Alvarez, G.A., Oliva, A., 2013. Real-world objects are not represented as bound units: Independent forgetting of different object details from visual memory. J. Exp. Psychol.: Gen. 142 (3), 791-808. doi:10.1037/a0029649.

Brass, M., Liefooghe, B., Braem, S., De Houwer, J., 2017. Following new task instructions: Evidence for a dissociation between knowing and doing. Neurosci Biobehav Rev 81 (June), 16-28. doi:10.1016/j.neubiorev.2017.02.012.

Brass, M., Wenke, D., Spengler, S., Waszak, F., 2009. Neural correlates of overcoming interference from instructed and implemented stimulus-response associations. J Neurosci 29 (6), 1766-1772. doi:10.1523/JNEUROSCI.5259-08.2009.

Brodeur, M.B., Guérard, K., Bouras, M., 2014. Bank of Standardized Stimuli (BOSS) Phase II: 930 New Normative Photos. PLoS One 9 (9), e106953. doi:10.1371/journal.pone. 0106953 .

Bundt, C., Bardi, L., Abrahamse, E.L., Brass, M., Notebaert, W., 2015. It wasn't me! Motor activation from irrelevant spatial information in the absence of a response. Front. Hum. Neurosci. 9 (OCT). doi:10.3389/fnhum.2015.00539.

Capilla, A., Schoffelen, J.-M., Paterson, G., Thut, G., Gross, J., 2014. Dissociated $\alpha$-Band modulations in the dorsal and ventral visual pathways in visuospatial attention and perception. Cereb. Cortex 24 (2), 550-561. doi:10.1093/cercor/bhs343.

Cavanagh, J.F., Frank, M.J., 2014. Frontal theta as a mechanism for cognitive control. Trends Cogn. Sci. 18 (8), 414-421. doi:10.1016/j.tics.2014.04.012.

Cheyne, D.O., 2013. MEG studies of sensorimotor rhythms: a review. Exp. Neurol. 245, 27-39. doi:10.1016/j.expneurol.2012.08.030.

Cohen, M.X., 2014a. Analyzing neural time series data: theory and practice. MIT Press.

Cohen, M. X. (2014b). Analyzing neural time series data.

Cohen, M.X., Donner, T.H., 2013. Midfrontal conflict-related theta-band power reflects neural oscillations that predict behavior. J. Neurophysiol. 110 (12), 2752-2763. doi:10.1152/jn.00479.2013.

Cole, M.W., Bagic, A., Kass, R., Schneider, W., 2010. Prefrontal dynamics underlying rapid instructed task learning reverse with practice. J. Neurosci. 30 (42), 14245-14254. doi:10.1523/JNEUROSCI.1662-10.2010.

Cole, M.W., Laurent, P., Stocco, A., 2013. Rapid instructed task learning: A new window into the human brain's unique capacity for flexible cognitive control. Cogn., Affect. Behav. Neurosci. 13 (1), 1-22. doi:10.3758/s13415-012-0125-7.

Combrisson, E., Perrone-Bertolotti, M., Soto, J.L., Alamian, G., Kahane, P., Lachaux, J.P., ... Jerbi, K., 2017. From intentions to actions: Neural oscillations encode motor processes through phase, amplitude and phase-amplitude coupling. Neuroimage 147, 473-487. doi:10.1016/j.neuroimage.2016.11.042.

Cooper, P.S., Karayanidis, F., McKewen, M., McLellan-Hall, S., Wong, A.S.W., Skippen, P., Cavanagh, J.F, 2019. Frontal theta predicts specific cognitive control-induced behavioural changes beyond general reaction time slowing. Neuroimage 189 (January), 130-140. doi:10.1016/j.neuroimage.2019.01.022.

de Vries, I.E.J., Slagter, H.A., Olivers, C.N.L, 2019. Oscillatory control over representational states in working memory. Trends Cogn. Sci. doi:10.1016/j.tics.2019.11.006.

de Vries, I.E.J., Van Driel, J., Karacaoglu, M., Olivers, C.N.L, 2018. Priority switches in visual working memory are supported by frontal delta and posterior alpha interactions. Cereb. Cortex 28 (11), 4090-4104. doi:10.1093/cercor/bhy223.

de Vries, I.E.J., van Driel, J., Olivers, C.N.L, 2019a. Decoding the status of working memory representations in preparation of visual selection. Neuroimage 191, 549-559. doi:10.1016/j.neuroimage.2019.02.069.

de Vries, I.E.J., van Driel, J., Olivers, C.N.L, 2019b. Decoding the status of working memory representations in preparation of visual selection. Neuroimage 191, 549-559. doi:10.1016/J.NEUROIMAGE.2019.02.069.

Delorme, A., Makeig, S., 2004. EEGLAB : an open source toolbox for analysis of single-trial EEG dynamics including independent component analysis. J. Neurosci Methods 134 9-21. doi:10.1016/j.jneumeth.2003.10.009.

Demanet, J., Liefooghe, B., Hartstra, E., Wenke, D., De Houwer, J., Brass, M., 2016. There is more into 'doing' than 'knowing': The function of the right inferior frontal sulcus is specific for implementing versus memorising verbal instructions. Neuroimage 141, 350-356. doi:10.1016/j.neuroimage.2016.07.059. 
Dumontheil, I., Thompson, R., Duncan, J., 2011. Assembly and use of new task rules in fronto-parietal cortex. J. Cogn. Neurosci. 23 (1), 168-182. doi:10.1162/jocn.2010.21439.

Duncan, J., Emslie, H., Williams, P., Johnson, R., Freer, C., 1996. Intelligence and the frontal lobe: the organization of goal-directed behavior. Cogn. Psychol. 30 (3), 257303. doi:10.1006/COGP.1996.0008.

Engel, A.K., Roelfsema, P.R., Fries, P., Brecht, M., Singer, W., 1997. Role of the temporal domain for response selection and perceptual binding. Cereb. Cortex 7 (6), 571-582. doi:10.1093/cercor/7.6.571.

Everaert, T., Theeuwes, M., Liefooghe, B., De Houwer, J., 2014. Automatic motor activation by mere instruction. Cogn., Affect. Behav. Neurosci. 14 (4), 1300-1309. doi:10.3758/s13415-014-0294-7.

Formica, S., González-García, C., Brass, M., 2020. The effects of declaratively maintaining and proactively proceduralizing novel stimulus-response mappings. Cognition 201, 104295. doi:10.1016/j.cognition.2020.104295.

Fries, P., 2005. A mechanism for cognitive dynamics: neuronal communication through neuronal coherence. Trends Cogn Sci 9 (10), 474-480. doi:10.1016/J.TICS.2005.08.011.

Fries, P., 2015, October 7. Rhythms for cognition: communication through coherence. Neuron. Cell Press doi:10.1016/j.neuron.2015.09.034.

Fukuda, K., Mance, I., Vogel, E.K., 2015. $\alpha$ power modulation and event-related slow wave provide dissociable correlates of visual working memory. J Neurosci 35 (41), 1400914016. doi:10.1523/JNEUROSCI.5003-14.2015.

González-García, C., Arco, J.E., Palenciano, A.F., Ramírez, J., Ruz, M., 2017. Encoding, preparation and implementation of novel complex verbal instructions. Neuroimage 148 (November 2016), 264-273. doi:10.1016/j.neuroimage.2017.01.037.

González-García, C., Formica, S., Liefooghe, B., Brass, M., 2020. Attentional prioritization reconfigures novel instructions into action-oriented task sets. Cognition 194, 104059. doi:10.1016/j.cognition.2019.104059.

González-García, C., Formica, S., Wisniewski, D., Brass, M., 2019. Frontoparietal action-oriented codes support novel task set implementation. BioRxiv, 830067 https://doi.org/10.1101/830067

Gould, I.C., Rushworth, M.F., Nobre, A.C., 2011. Indexing the graded allocation of visuospatial attention using anticipatory alpha oscillations. J Neurophysiol 105 (3), 13181326. doi:10.1152/jn.00653.2010.

Goyal, A., Miller, J., Qasim, S., Watrous, A.J., Stein, J.M., Inman, C.S., ... Jacobs, J., 2020. Functionally distinct high and low theta oscillations in the human hippocampus. BioRxiv, 498055 doi:10.1101/498055.

Griffin, G., Holub, A., \& Perona, P. (2007). Caltech-256 Object Category Dataset. Retrieved from https://authors.library.caltech.edu/7694/

Groppe, D.M., Makeig, S., Kutas, M., 2009. Identifying reliable independent components via split-half comparisons. Neuroimage 45 (4), 1199-1211. doi:10.1016/j.neuroimage.2008.12.038.

Haller, M., Donoghue, T., Peterson, E., Varma, P., Sebastian, P., Gao, R., ... Voytek, B., 2018. Parameterizing neural power spectra. Bio Rxiv, 299859 doi:10.1101/299859.

Hari, R., Salmelin, R., 1997, January 1 . Human cortical oscillations: a neuromagnetic view through the skull. Trends Neurosci doi:10.1016/S0166-2236(96)10065-5.

Hartstra, E., Kühn, S., Verguts, T., Brass, M., 2011. The implementation of verbal instructions: an fMRI study. Hum. Brain Mapp. 32 (11), 1811-1824. doi:10.1002/hbm.21152.

Hartstra, E., Waszak, F., Brass, M., 2012. The implementation of verbal instructions: Dissociating motor preparation from the formation of stimulus-response associations. Neuroimage 63 (3), 1143-1153. doi:10.1016/j.neuroimage.2012.08.003.

Itthipuripat, S., Wessel, J.R., Aron, A.R., 2013. Frontal theta is a signature of successful working memory manipulation. Exp. Brain Res. 224 (2), 255-262. doi:10.1007/s00221-012-3305-3.

Jasp Team. (2019). JASP (Version 0.11.1)[Computer software]. Retrieved from https://jasp-stats.org/

Jensen, O., 2002. Oscillations in the Alpha Band (9-12 Hz) Increase with Memory Load during Retention in a Short-term Memory Task. Cereb. Cortex 12 (8), 877-882. doi:10.1093/cercor/12.8.877.

Jensen, O., Mazaheri, A., 2010. Shaping Functional Architecture by Oscillatory Alpha Activity: Gating by Inhibition. Frontiers in Human Neuroscience 4 (November), 1-8. doi:10.3389/fnhum.2010.00186.

Keefe, J.M., Störmer, V.S., 2021. Lateralized alpha activity and slow potential shifts over visual cortex track the time course of both endogenous and exogenous orienting of attention. Neuroimage 225, 117495. doi:10.1016/j.neuroimage.2020.117495.

Klem, G.H., Lüders, H.O., Jasper, H.H., Elger, C., 1999. The ten-twenty electrode system of the International Federation. The International Federation of Clinical Neurophysiology. Electroencephalogr. Clin. Neurophysiol.. Suppl. 52, 3-6.

Klimesch, W., Sauseng, P., Hanslmayr, S., 2007. EEG alpha oscillations: the inhibition-timing hypothesis. Brain Res. Rev 53 (1), 63-88. doi:10.1016/J.BRAINRESREV.2006.06.003.

Konkle, T., Brady, T.F., Alvarez, G.A., Oliva, A., 2010. Conceptual distinctiveness supports detailed visual long-term memory for real-world objects. J. Exp. Psychol.: Gen. 139 (3), 558-578. doi:10.1037/a0019165.

Lakatos, P., Gross, J., Thut, G., 2019, September 23. A new unifying account of the roles of neuronal entrainment. Current Biology. Cell Press doi:10.1016/j.cub.2019.07.075.

Liefooghe, B., De Houwer, J., 2017. Automatic effects of instructions do not require the intention to execute these instructions. J. Cogn. Psychol. 30 (1), 108-121. doi:10.1080/20445911.2017.1365871.

Liefooghe, B., Wenke, D., De Houwer, J., 2012. Instruction-based task-rule congruency effects. J. Exp. Psychol.: Learn., Memory, Cogn. 38 (5), 1325-1335. doi: $10.1037 / \mathrm{a} 0028148$.

Lisman, J.E., Jensen, O., 2013. The theta-gamma neural code. Neuron 77 (6), 1002-1016. doi:10.1016/j.neuron.2013.03.007.
Manza, P., Hau, C.L.V., Leung, H.-C, 2014. Alpha power gates relevant information during working memory updating. J. Neurosci. 34 (17), 5998-6002. doi:10.1523/JNEUROSCI.4641-13.2014.

Marchesotti, S., Bassolino, M., Serino, A., Bleuler, H., Blanke, O., 2016. Quantifying the role of motor imagery in brain-machine interfaces. Sci. Rep. 6 (1), 24076. doi:10.1038/srep24076.

Maris, E., Oostenveld, R., 2007. Nonparametric statistical testing of EEG- and MEG-data J. Neurosci. Methods 164, 177-190. doi:10.1016/j.jneumeth.2007.03.024.

Mazaheri, A., van Schouwenburg, M.R., Dimitrijevic, A., Denys, D., Cools, R., Jensen, O., 2014. Region-specific modulations in oscillatory alpha activity serve to facilitate processing in the visual and auditory modalities. Neuroimage 87, 356-362. doi:10.1016/J.NEUROIMAGE.2013.10.052.

McFarland, D.J., Miner, L.A., Vaughan, T.M., Wolpaw, J.R., 2000. Mu and Beta Rhythm Topographies During Motor Imagery and Actual Movements. Brain Topogr. 12 (3), 177-186. doi:10.1023/A:1023437823106.

McLelland, D., VanRullen, R., 2016. Theta-Gamma Coding Meets Communication-through-Coherence: Neuronal Oscillatory Multiplexing Theories Reconciled. PLoS Comput. Biol. 12 (10), e1005162.

Meiran, N., Pereg, M., Kessler, Y., Cole, M.W., Braver, T.S., 2015a. Reflexive activation of newly instructed stimulus-response rules: evidence from lateralized readiness potentials in no-go trials. Cogn., Affect. Behav. Neurosci. 15 (2), 365-373. doi:10.3758/s13415-014-0321-8.

Meiran, N., Pereg, M., Kessler, Y., Cole, M.W., Braver, T.S., 2015b. The power of instructions: Proactive configuration of stimulus-response translation. J. Exp. Psychol.: Learn., Memory, Cogn. 41 (3), 768-786. doi:10.1037/xlm0000063.

Milner, B., 1963. Effects of different brain lesions on card sorting: the role of the frontal lobes. Arch Neurol 9 (1), 90-100. doi:10.1001/archneur.1963.00460070100010.

Mok, R.M., Myers, N.E., Wallis, G., Nobre, A.C., 2016. Behavioral and neural markers of flexible attention over working memory in aging. Cereb. Cortex 26 (4), 1831-1842. doi:10.1093/cercor/bhw011.

Muhle-Karbe, P.S., Duncan, J., De Baene, W., Mitchell, D.J., Brass, M., 2017. Neural coding for instruction-based task sets in human frontoparietal and visual cortex. Cereb. Cortex (New York, N.Y. : 1991) 27 (3), 1891-1905. doi:10.1093/cercor/bhw032.

Myers, N.E., Stokes, M.G., Nobre, A.C., 2017. Prioritizing information during working memory: beyond sustained internal attention. Trends Cogn. Sci. 21 (6), 449-461. doi:10.1016/j.tics.2017.03.010.

Myers, N.E., Walther, L., Wallis, G., Stokes, M.G., Nobre, A.C., 2015. Temporal dynamics of attention during encoding versus maintenance of working memory: complementary views from event-related potentials and alpha-band oscillations. J. Cogn. Neurosci. 27 (3), 492-508. doi:10.1162/jocn_a_00727.

Neuper, C., Pfurtscheller, G., 2001. Event-related dynamics of cortical rhythms: Frequency-specific features and functional correlates. Int. J. Psychophysiol. 43 (1), 41-58. doi:10.1016/S0167-8760(01)00178-7.

Nobre, A.C., Van Ede, F., 2018. Anticipated moments: Temporal structure in attention. Nat. Rev. Neurosci. 19 (1), 34-48. doi:10.1038/nrn.2017.141.

Olivers, C.N.L., Eimer, M, 2011. On the difference between working memory and attentional set. Neuropsychologia 49 (6), 1553-1558. doi:10.1016/j.neuropsychologia.2010.11.033.

Olivers, C.N.L., Peters, J., Houtkamp, R., Roelfsema, P.R, 2011, July. Different states in visual working memory: when it guides attention and when it does not. Trends Cogn. Sci. doi:10.1016/j.tics.2011.05.004.

Onton, J., Delorme, A., Makeig, S., 2005. Frontal midline EEG dynamics during working memory. Neuroimage 27 (2), 341-356. doi:10.1016/J.NEUROIMAGE.2005.04.014.

Oostenveld, R., Fries, P., Maris, E., Schoffelen, J.-M., 2010. FieldTrip: Open Source Software for Advanced Analysis of MEG, EEG, and Invasive Electrophysiological Data. Comput. Intell. Neurosci., 2011 doi:10.1155/2011/156869.

Palenciano, A.F., González-García, C., Arco, J.E., Pessoa, L., Ruz, M., 2019. Representational organization of novel task sets during proactive encoding. J. Neurosci. doi:10.1523/jneurosci.0725-19.2019, 0725-19.

Palenciano, A.F., González-García, C., Arco, J.E., Ruz, M., 2019. Transient and sustained control mechanisms supporting novel instructed behavior. Cereb. Cortex 29 (9), 39483960. doi:10.1093/cercor/bhy273.

Perrin, F., Pernier, J., Bertrand, O., Echallier, J.F., 1989. Spherical splines for scalp potential and current density mapping. Electroencephalogr. Clin. Neurophysiol. 72 (2), 184-187. doi:10.1016/0013-4694(89)90180-6.

Pfurtscheller, G., Brunner, C., Schlögl, A., Lopes da Silva, F.H., 2006. Mu rhythm (de)synchronization and EEG single-trial classification of different motor imagery tasks. Neuroimage 31 (1), 153-159. doi:10.1016/J.NEUROIMAGE.2005.12.003.

Pfurtscheller, G., Neuper, C., 1997. Motor imagery activates primary sensorimotor area in humans. Neurosci. Lett. 239 (2-3), 65-68. doi:10.1016/S0304-3940(97)00889-6.

Pineda, J.A., 2005. The functional significance of mu rhythms: translating 'seeing' and 'hearing' into 'doing. Brain Res. Rev. 50 (1), 57-68. doi:10.1016/j.brainresrev.2005.04.005.

Poch, C., Campo, P., Barnes, G.R., 2014. Modulation of alpha and gamma oscillations related to retrospectively orienting attention within working memory. Eur. J. Neurosci. 40 (2), 2399-2405. doi:10.1111/ejn.12589.

Poch, C., Carretie, L., Campo, P., 2017. A dual mechanism underlying alpha lateralization in attentional orienting to mental representation. Biol. Psychol. 128 (March), 63-70. doi:10.1016/j.biopsycho.2017.07.015.

Poch, C., Valdivia, M., Capilla, A., Hinojosa, J.A., Campo, P., 2018. Suppression of nolonger relevant information in Working Memory: an alpha-power related mechanism? Biol. Psychol. 135 (February), 112-116. doi:10.1016/j.biopsycho.2018.03.009.

Popov, T., Popova, P., Harkotte, M., Awiszus, B., Rockstroh, B., Miller, G.A., 2018. Cross-frequency interactions between frontal theta and posterior alpha control mechanisms foster working memory. Neuroimage 181 (July), 728-733. doi:10.1016/j.neuroimage.2018.07.067. 
Rhodes, E., Gaetz, W.C., Marsden, J., Hall, S.D., 2018. Transient Alpha and Beta Synchrony underlies preparatory recruitment of directional motor networks. J. Cogn. Neurosci. 30 (6), 867-875. doi:10.1162/jocn a 01250.

Riddle, J., Scimeca, J.M., Cellier, D., Dhanani, S., Esposito, M.D., Riddle, J., ... Esposito, M.D., 2020. Causal evidence for a role of theta and alpha oscillations in the control of working memory. Curr. Biol. 1-7. doi:10.1016/j.cub.2020.02.065.

Rihs, T.A., Michel, C.M., Thut, G., 2007. Mechanisms of selective inhibition in visual spatial attention are indexed by $\alpha$-band EEG synchronization. Eur. J. Neurosci. 25 (2), 603-610. doi:10.1111/j.1460-9568.2007.05278.x.

Rihs, T.A., Michel, C.M., Thut, G., 2009. A bias for posterior $\alpha$-band power suppression versus enhancement during shifting versus maintenance of spatial attention. Neuroimage 44 (1), 190-199. doi:10.1016/j.neuroimage.2008.08.022.

Rohenkohl, G., Nobre, A.C., 2011. Alpha oscillations related to anticipatory attention follow temporal expectations. J. Neurosci. 31 (40), 14076-14084. doi:10.1523/JNEUROSCI.3387-11.2011.

Ruge, H., Schäfer, T.A., Zwosta, K., Mohr, H., Wolfensteller, U., 2019. Neural representation of newly instructed rule identities during early implementation trials. ELife 8, 1-25. doi:10.7554/eLife.48293.

Ruge, H., Wolfensteller, U., 2010. Rapid formation of pragmatic rule representations in the human brain during instruction-based learning. Cereb. Cortex 20 (7), 1656-1667. doi:10.1093/cercor/bhp228.

Sassenhagen, J., Draschkow, D., 2019. Cluster-based permutation tests of MEG/EEG data do not establish significance of effect latency or location. Psychophysiology (August 2018) 1-8. doi:10.1111/psyp.13335.

Sauseng, P., Klimesch, W., Stadler, W., Schabus, M., Doppelmayr, M., Hanslmayr, S., ... Birbaumer, N., 2005. A shift of visual spatial attention is selectively associated with human EEG alpha activity. Eur. J. Neurosci. 22 (11), 2917-2926. doi:10.1111/j.1460-9568.2005.04482.x.

Schneider, D., Barth, A., Wascher, E., 2017. On the contribution of motor planning to the retroactive cuing benefit in working memory: evidence by mu and beta oscillatory activity in the EEG. Neuroimage 162 (April), 73-85. doi:10.1016/j.neuroimage.2017.08.057.

Schneider, D., Mertes, C., Wascher, E., 2016. The time course of visuo-spatial working memory updating revealed by a retro-cuing paradigm. Sci. Rep. 6 (1), 21442. doi:10.1038/srep21442.

Senoussi, M., Moreland, J.C., Busch, N.A., Dugué, L., 2019. Attention explores space periodically at the theta frequency. J. Vis. 19 (5), 1-17. doi:10.1167/19.5.22.

Senoussi, M., Verbeke, P., Desender, K., De Loof, E., Talsma, D., Verguts, T., Dunantlaan, H., 2020. Theta oscillations shift towards optimal frequency for cognitive control. BioRxiv doi:10.1101/2020.08.30.273706, 2020.08.30.273706.

Souza, A.S., Oberauer, K., 2016. In search of the focus of attention in working memory: 13 years of the retro-cue effect. Atten., Percept. Psychophys. 78 (7), 1839-1860. doi:10.3758/s13414-016-1108-5.

Thut, G., Nietzel, A., Brandt, S.A., Pascual-Leone, A, 2006. Alpha-band electroencephalographic activity over occipital cortex indexes visuospatial attention bias and predicts visual target detection. J. Neurosci. : Off. J. Soc. Neurosci. 26 (37), 9494-9502. doi:10.1523/JNEUROSCI.0875-06.2006.

Thut, G., Schyns, P.G., Gross, J., 2011. Entrainment of perceptually relevant brain oscillations by non-invasive rhythmic stimulation of the human brain. Front. Psychol. 2 (JUL), 170. doi:10.3389/fpsyg.2011.00170.
Thut, G., Veniero, D., Romei, V., Miniussi, C., Schyns, P., Gross, J., 2011. Rhythmic TMS causes local entrainment of natural oscillatory signatures. Curr. Biol. 21 (14), 11761185. doi:10.1016/j.cub.2011.05.049.

Tzagarakis, C., West, S., Pellizzer, G., 2015. Brain oscillatory activity during motor preparation: Effect of directional uncertainty on beta, but not alpha, frequency band. Front. Neurosci. 9 (JUN), 1-13. doi:10.3389/fnins.2015.00246.

Van Diepen, R., Foxe, J.J., Mazaheri, A., 2019. The functional role of alpha-band activity in attentional processing: The current zeitgeist and future outlook. Curr. Opin. Psychol. doi:10.1016/J.COPSYC.2019.03.015.

van Dijk, H., Schoffelen, J.-M., Oostenveld, R., Jensen, O., 2008. Prestimulus oscillatory activity in the alpha band predicts visual discrimination ability. J. Neurosci. : Off. J. Soc. Neurosci. 28 (8), 1816-1823. doi:10.1523/JNEUROSCI.1853-07.2008.

van Ede, F., 2017. Mnemonic and attentional roles for states of attenuated alpha oscillations in perceptual working memory: a review. Eur. J. Neurosci. 1-7. doi:10.1111/ejn.13759.

van Ede, F., 2020. Visual working memory and action: functional links and bi-directional influences. Vis. Cogn. 1-13. doi:10.1080/13506285.2020.1759744.

van Ede, F., Chekroud, S.R., Stokes, M.G., Nobre, A.C., 2019a. Concurrent visual and motor selection during visual working memory guided action. Nat. Neurosci. 22 (3), 477483. doi:10.1038/s41593-018-0335-6.

van Ede, F., Chekroud, S.R., Stokes, M.G., Nobre, A.C., 2019b. Concurrent visual and motor selection during visual working memory guided action. Nat. Neurosci. 22 (3), 477-483. doi:10.1038/s41593-018-0335-6.

Van Elswijk, G., Kleine, B.U., Overeem, S., Stegeman, D.F., 2007. Expectancy induces dynamic modulation of corticospinal excitability. J. Cogn. Neurosci. 19 (1), 121-131. doi:10.1162/jocn.2007.19.1.121.

Verbeke, P., Verguts, T., 2019. Learning to synchronize: how biological agents can couple neural task modules for dealing with the stability-plasticity dilemma. PLoS Comput. Biol. 15 (8), e1006604. doi:10.1371/journal.pcbi.1006604.

Verguts, T., 2017. Binding by random bursts: A computational model of cognitive control. J. Cogn. Neurosci. doi:10.1162/jocn.

Wallis, G., Stokes, M., Cousijn, H., Woolrich, M., Nobre, A.C., 2015. Frontoparietal and Cingulo-opercular Networks Play Dissociable Roles in Control of Working Memory. J. Cogn. Neurosci. 27 (10), 2019-2034. doi:10.1162/jocn a 00838.

Walter, W.G., Cooper, R., Aldridge, V.J., McCallum, W.C., Winter, A.L., 1964. Contingent negative variation : An electric sign of sensori-motor association and expectancy in the human brain. Nature 203 (4943), 380-384. doi:10.1038/203380a0.

Wenke, D., Gaschler, R., Nattkemper, D., Frensch, P.A., 2009. Strategic influences on implementing instructions for future actions. Psychol. Res. Psychologische Forschung 73 (4), 587-601. doi:10.1007/s00426-009-0239-x.

Wiener, M., Parikh, A., Krakow, A., Coslett, H.B., 2018. An intrinsic role of beta oscillations in memory for time estimation. Sci. Rep. 8 (1), 1-17. doi:10.1038/s41598-018-26385-6.

Wolff, M.J., Ding, J., Myers, N.E., Stokes, M.G., 2015. Revealing hidden states in visual working memory using electroencephalography. Front. Syst. Neurosci. 9 (September), 1-12. doi:10.3389/fnsys.2015.00123.

Wolff, M.J., Jochim, J., Akyürek, E.G., Stokes, M.G., 2017. Dynamic hidden states underlying working-memory-guided behavior. Nat. Neurosci. 20 (6), 864-871. doi: $10.1038 / \mathrm{nn} .4546$. 